\title{
Performance of Trajectory Models with Wind Uncertainty
}

\author{
Alan G. Lee* \\ NASA Ames Research Center MS 210-8, Moffett Field, CA, 94035 \\ Stephen S. Weygandt, Ph.D. ${ }^{\dagger}$ \\ NOAA/FSL, R/FS1 325 Broadway, Boulder, CO, 80305-3328 \\ Barry Schwartz ${ }^{\ddagger}$ \\ Scientific Research Group 325 Broadway, Boulder, CO, 80305-3328 \\ and \\ James R. Murphy ${ }^{\S}$ \\ NASA Ames Research Center MS 210-8, Moffett Field, CA, 94035
}

\begin{abstract}
Typical aircraft trajectory predictors use wind forecasts but do not account for the forecast uncertainty. A method for generating estimates of wind prediction uncertainty is described and its effect on aircraft trajectory prediction uncertainty is investigated. The procedure for estimating the wind prediction uncertainty relies uses a time-lagged ensemble of weather model forecasts from the hourly updated Rapid Update Cycle (RUC) weather prediction system. Forecast uncertainty is estimated using measures of the spread amongst various RUC time-lagged ensemble forecasts. This proof of concept study illustrates the estimated uncertainty and the actual wind errors, and documents the validity of the assumed ensemble-forecast accuracy relationship. Aircraft trajectory predictions are made using RUC winds with provision for the estimated uncertainty. Results for a set of simulated flights indicate this simple approach effectively translates the wind uncertainty estimate into an aircraft trajectory uncertainty. A key strength of the method is the ability to relate uncertainty to specific weather phenomena (contained in the various ensemble members) allowing identification of regional variations in uncertainty.
\end{abstract}

\section{Introduction}

A ir Traffic Control (ATC) decision support tools (DST) are designed to help air traffic controllers with the management of the aircraft into and through the en route airspace. These tools provide conflict detection and resolution and scheduling guidance and advanced scheduling algorithms such as Direct To (D2) and Traffic Management Advisor (TMA). ${ }^{1}$ Common to each of these tools is the need for accurate aircraft trajectories. Elements of trajectory prediction errors can come from the following sources: aircraft surveillance (radar, ADS-B), intent (controller instruction, pilot procedures), navigation, aircraft performance modeling, and weather forecasts. Many air traffic control decision support tools such as Center TRACON Automation System (CTAS) use Rapid Update Cycle (RUC) wind data as an input to their aircraft trajectory predictors. To better understand the wind forecast errors, several studies investigated the accuracy of the RUC data and aircraft trajectory uncertainty. ${ }^{2,3,4} \mathrm{~A}$ few studies also looked at the impact of more accurate wind data on the resultant trajectory., ${ }^{5,}$ This previous work

\footnotetext{
* Aerospace Engineer, Aerospace Simulation Research and Development Branch, MS 210-8, AIAA Member.

${ }^{\dagger}$ Assimilation Development Section Chief, Assimilation and Modeling Branch, 325 Broadway, AIAA Member.

$\ddagger$ Contractor, AIAA Non-Member.

$\S$ Software Manager, Aerospace Simulation Research and Development Branch, MS 210-8, AIAA Senior Member. 
focused on the average accuracy of the wind forecast. Forecasted wind errors are not, however, provided by the RUC developers for use in ATC DSTs.

Past work on the subject of trajectory prediction uncertainty includes a study by D. Thipphavong ${ }^{7}$ that focused on the influence of aircraft weight as a source of trajectory prediction uncertainty. More relevant to this paper is a study performed by S. Mondoloni ${ }^{8}$ that examined wind prediction uncertainty as a source of trajectory prediction uncertainty using a statistical wind prediction uncertainty model.

This paper describes a method to compute RUC wind uncertainty and how these uncertainties affect CTAS aircraft trajectory predictions. CTAS is used to estimate the spread of trajectory predictions due to wind uncertainty. Finally, the paper quantifies the variability in the resultant trajectories and discusses the impact of that variability on CTAS tools.

\section{Background}

\section{A. RUC Forecasts and Forecast Uncertainty}

The aviation community relies on the National Oceanic and Atmospheric Administration RUC $^{9,10}$ wind forecasts heavily. It is widely used by the Federal Aviation Administration (FAA) in various decision support tools ${ }^{11,12}$. A particular focus is on upper-level wind forecast accuracy. The RUC produces highly accurate upper-level wind forecasts for the Continental United States ${ }^{13}$ by applying all available weather observations as a fresh set of initial conditions to their weather model Gridded RUC wind fields (from 13-km resolution 12-hour forecasts) provide 1-8 hour forecasts of wind speed (magnitude) and direction, and atmospheric pressure at 40-km horizontal intervals and 50 vertical levels (variable vertical resolution).

The RUC has been extensively verified against rawinsonde, profiler, aircraft, surface, and radar observations, providing a documentation of average RUC errors, as well as the variability in RUC Skill amongst different forecasts. ${ }^{10,14}$ Skill refers to a statistical evaluation of the accuracy of forecasts. In addition to the variability in RUC Skill between given forecasts, regional variability exists across the area within each RUC forecast. Thus, for many users who depend critically on the accuracy of RUC upper-level winds, there is a strong interest in methods for estimating a priori the RUC at a specific location. Of course, the actual forecast error can only be assessed relative to observed data (which have their own errors) after the observations are collected at the forecast valid time. Thus, we distinguish between forecast errors (assessed relative to observed data after the forecast valid time), and forecast uncertainty, which is an estimate of the expected forecast error for a specific forecast.

The work in this paper uses a technique for generating wind uncertainty fields based on an assumption that the magnitude of the variability amongst members of a time-lagged ensemble ${ }^{15}$ of RUC forecasts is proportional to the likely magnitude of the forecast error. The time-lagged ensemble consists of several successive RUC forecasts of different lengths that are all valid at the same time. For this application 2, 3, 4, 5,6 and 7-hour RUC wind forecasts are used to construct the time-lagged ensemble. It should be noted that many methods for generating forecast ensembles exist (including techniques in which the initial conditions and / or model physics are perturbed), but most are very computationally intensive and time-consuming, requiring many different model runs for a given forecast. Because a new model forecast is created each hour as part of the RUC system, it is ideally suited to this time-lagged technique approach where the ensemble requires no additional model forecasts. Two disadvantages of the timelagged ensemble approach, however, are: 1) the older forecasts usually have larger errors; and 2) the correlation between different ensemble members is typically larger than for other techniques. Using the time-lagged ensemble approach and assuming a Gaussian distribution, a wind uncertainty of one standard deviation is produced for both the $U$ and the $V$ wind components (increasing Eastward along the $U$ axis and Northward along the $V$ axis). Since the wind uncertainty is applied to the $\mathrm{U}$ and $\mathrm{V}$ components of the wind field vector, it will have impacts on the trajectories when there is a change in the direction of the forecasted wind or the magnitude of the wind vector.

\section{B. Trajectory Generation}

The Center TRACON Automation System (CTAS) Trajectory Synthesizer (TS) software was used to generate the trajectories for the analysis in this paper. CTAS is a collection of programs developed by the National Aeronautics and Space Administration (NASA) to support Air Traffic Management (ATM) research. These programs, including a route parser, scheduler, and the TS, form the basis for ATM tools such as the Traffic Management Advisor (TMA) and the En Route Descent Advisor (EDA). ${ }^{16}$ To generate a trajectory prediction, CTAS first applies route parsing to the flight plan route (supplied by the Host or other data source) and sends the intended horizontal path and vertical constraints, along with the current state information to the trajectory predictor (TS). The TS uses a point-mass aircraft equation-of-motion model that incorporates winds, atmospheric data, and specific aircraft performance information, such as thrust and drag. The model is described in detail by Slattery, et 
al. ${ }^{17}$ The RUC data is interpolated to compute the wind speed, direction, and atmospheric pressure at any given point in the airspace.

Wind data is a component for two major aspects of trajectory calculation. The first aspect is for computing the aircraft's intended Mach or calibrated airspeed (CAS). If an aircraft is at cruise altitude, many decision support tools use the current wind field and the ground speed to calculate a true airspeed (TAS). This is shown in Equation 1. The intended airspeed is then calculated in terms of a Mach or CAS and is typically fixed for the cruise prediction. The second aspect is that, given an intended airspeed, either provided in a flight plan or derived as described above, the wind along the predicted path is used to determine the relative ground speed for that segment of flight.

$$
\vec{V}_{T A S}=\vec{V}_{\text {ground }}-\vec{V}_{\text {wind }}
$$

Wind data in trajectory prediction, therefore, ultimately alters the intended speed of the aircraft relative to the ground. This, in turn, affects the predicted position of the aircraft along its modeled track. Depending on the intended use of the trajectory, this can be interpreted in differently: either as an along track position difference or a difference of time at a given point. For ATM purposes the variation of along track position can be used to determine a conflict separation buffer. Alternately, differences in time of flight to a particular waypoint can be used for ATM scheduling.

\section{Methodology}

\section{A. Weather Data Files}

Using standard output from six successive RUC model predictions $\left(2,3,4,5,6\right.$, and 7-hr), all valid for April $3^{\text {rd }}$ 2009, 0 Zulu time, three different RUC datasets were created: 1) standard forecasts, 2) an ensemble average 3) the 0$\mathrm{hr}$ Analysis forecast, and 4) a prediction with the wind uncertainty applied. The standard forecast output formed the members of the ensemble. The average $U$ and $V$ component of the wind for the forecast ensemble members at every point was defined as the ensemble average. The standard deviation of the $U$ and $V$ components among the ensemble members was defined as the wind uncertainty. The $U$ and $V$ uncertainty magnitudes (one standard deviation) were applied in predefined cardinal directions to a base forecast taken as either the ensemble average or the 2-hr forecast. Therefore for each base forecast, 4 files were created whose $U$ and $V$ component wind magnitudes were $U_{\text {base }} \pm$ $U_{\text {uncert }}$ and $V_{\text {base }} \pm V_{\text {uncert. }}$ Where $U_{\text {base }}$ and $U_{\text {uncert }}$ are the $U$ component wind speeds of the base forecast and uncertainty magnitudes respectively (and similarly for the $\mathrm{V}$ component). Since these uncertainty values were applied in a predefined manner, the aircraft were restricted to fly a constant heading using these files. This approach, while not ideal, was the most expedient implementation.

\section{B. Trajectory Data Collection}

Since the study did not measure the trajectory predictions against an actual flight track, fabricated flight trajectories were created to study various atmospheric patterns of interest. All flights used a Boeing 767 aircraft model in en-route cruise flight at a constant altitude, heading and speed. Each aircraft flew at an altitude of 34,000 feet and speed of 0.80 mach.

The first portion of the study examined only the $U$ component wind uncertainty and the aircraft were assigned a due west heading. CTAS was used to generate six hypothetical flight cases with each flight case using the RUC wind forecast files described previously. Figures $2 a$ and $2 b$ show the flight path of each aircraft relative to the $U$ and $\mathrm{V}$ component wind uncertainty at FL340. The second part of the study investigated the interaction of the U and V components. 


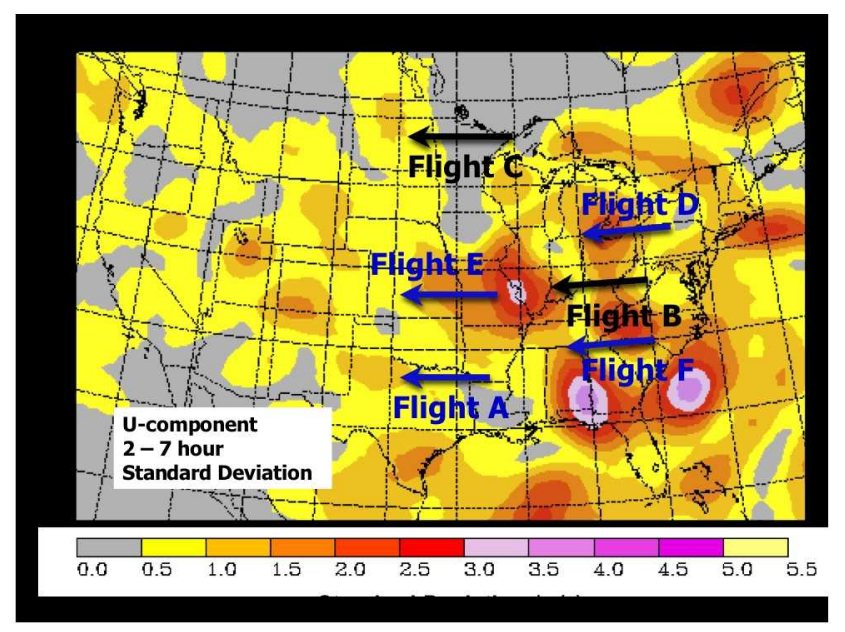

Figure 2a: Modeled Flight Paths in Relation to the UComponent Wind Uncertainty used in this Study at FL340

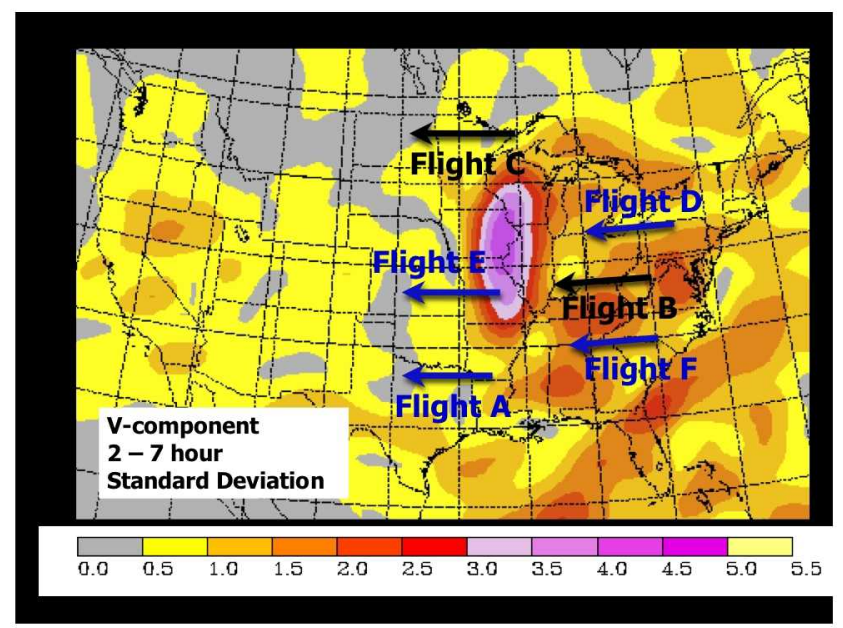

Figure 2b: Modeled Flight Paths in Relation to the V-Component Wind Uncertainty used in this Study at FL340

\section{Data Analysis}

\section{A. Analysis of Time Lagged Wind Forecast Uncertainty Model}

Prior to examining the use of the time-lagged ensemble technique for estimating aircraft trajectory uncertainty, the forecast errors of the various RUC time-lagged forecasts and the uncertainty estimate obtained from the timelagged ensemble members must be assessed. First, the upper-level wind error is computed as a function of forecast length over a long time period, and then forecast error scores are examined from a single case study verification time $(00 \mathrm{z} 3$ April, 2009) for both total wind and the U component. Next, the correlation between the wind forecast uncertainty (as measured by the standard deviation of the ensemble members) with the forecast error (as measured by the forecast - 0-hr Analysis forecast difference) is presented. Establishing the validity of this assumed relationship between the magnitudes of the ensemble spread and the forecast error is crucial for establishing the utility using the time-lagged ensemble to generate wind uncertainty fields.

\section{Wind forecast accuracy vs. observations and analyses}

Fig. 3 shows vertical profiles of RUC upper level vector wind root mean square (RMS) errors verified against rawinsondes observations for different forecast lengths (1, 2, 3, 6, and 9-hr) and the 0-hr Analysis forecast. Altitudes are labeled in millibars of pressure $(700-200 \mathrm{mb}$, or $9878-38615 \mathrm{feet})$ on the vertical axis. The RMS error profiles of each forecast length are computed from a long-term (four month) average. Readily apparent in the figure is the consistent decrease in upper level wind errors as forecast length is decreased. Note that the latency to generate the 1-hr forecast is too great to be used by CTAS for real-time trajectory prediction.. Also evident in the figure is the "error" of the 0-hr Analysis forecast, which varies from about $2.7 \mathrm{~m} / \mathrm{s}$ at $600 \mathrm{mb}$ (13794 feet) to $3.5 \mathrm{~m} / \mathrm{s}$ above $300 \mathrm{mb}$ (30052 feet). The 0-hr Analysis forecast blends new observed data with a gridded prior estimate provided by the previous 1-h RUC forecast. The 0-hr Analysis forecast does not perfectly match the observations for two reasons: 1) the observations contain errors, and; 2) the observations include contributions from length-scales too small to be represented in the model (termed errors of representativeness). With an understanding of the relationship between errors relative to the 0 -hr Analysis forecast and relative to the observations, this study uses the 0 -hr Analysis forecast for comparison of errors, as it allows for easier examination of spatial aspects. It is important, however, to document the correspondence between observation verification and 0-hr Analysis forecast verification, which we will do for the selected test case period. 


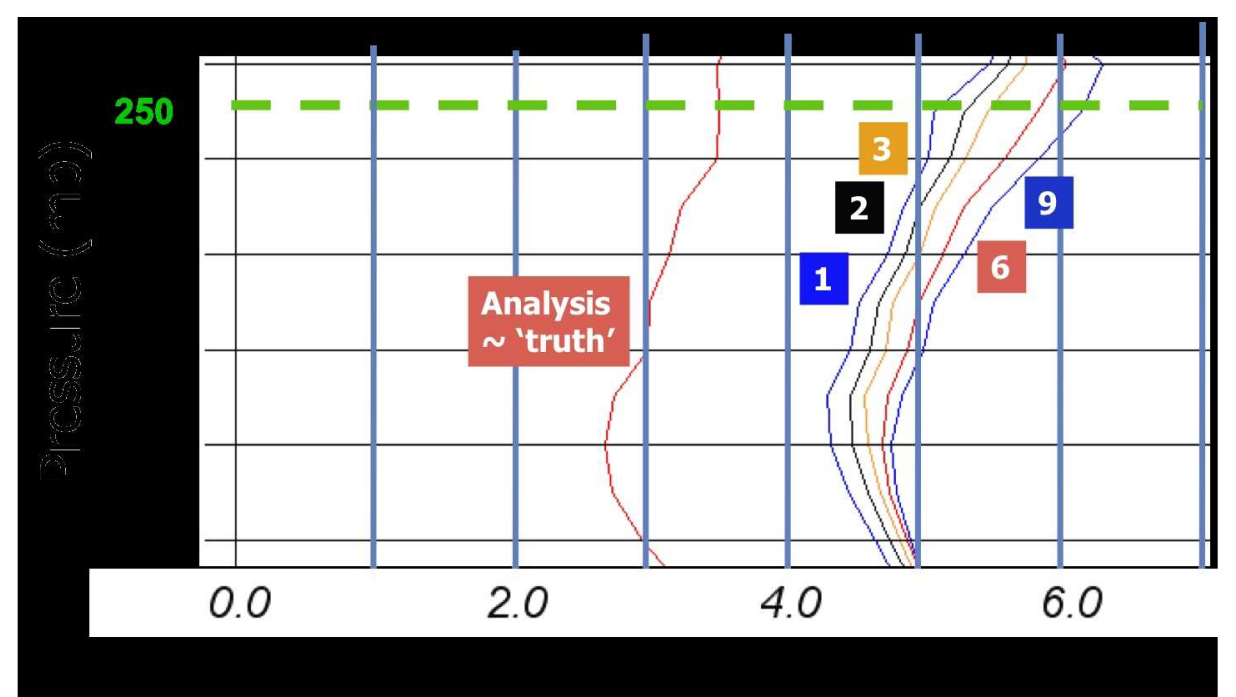

Figure 3: RUC upper level vector wind root mean square (RMS) errors verified against rawinsondes for 0-hr Analysis forecast and different forecast lengths (1, 2, 3, 6, and 9 hour)

Fig. 4 shows the $250 \mathrm{mb}$ (FL 340) forecast wind RMS errors for different forecast lead times all valid at 0000 UTC on 3 April, 2009, for comparison with both the rawinsonde observations (red curve, scale on left) and the 0-hr Analysis forecast (solid blue curve, scale on right). The mean absolute errors (MAE) relative to the 0-hr Analysis forecast (scale on the right) of the forecast U-component are shown by the blue dotted line. Both RMS errors and MAEs are shown because RMS errors are typically used for long term RUC skill statistics, but we have used MAEs to compute the uncertainty. A number of features are readily apparent in the plot. First, comparison with the rawinsonde network shows larger $250 \mathrm{mb}$ wind errors for this day than for the four month average (compare with the values along the green dotted curve in Fig. 3), indicating this day to have above average forecast error. Second, comparison of the vector wind RMS error for the observation verification (red solid curve, scale on the left) with that for the 0-hr Analysis verification (solid blue curve, scale on the right) indicates a high degree of consistency between the two verification measures. Lastly, as expected, the MAE for the U-component of the wind is smaller than the vector wind RMS, but shows a similar increase as forecast lead-time is increased (note all these forecasts are valid at the same time).

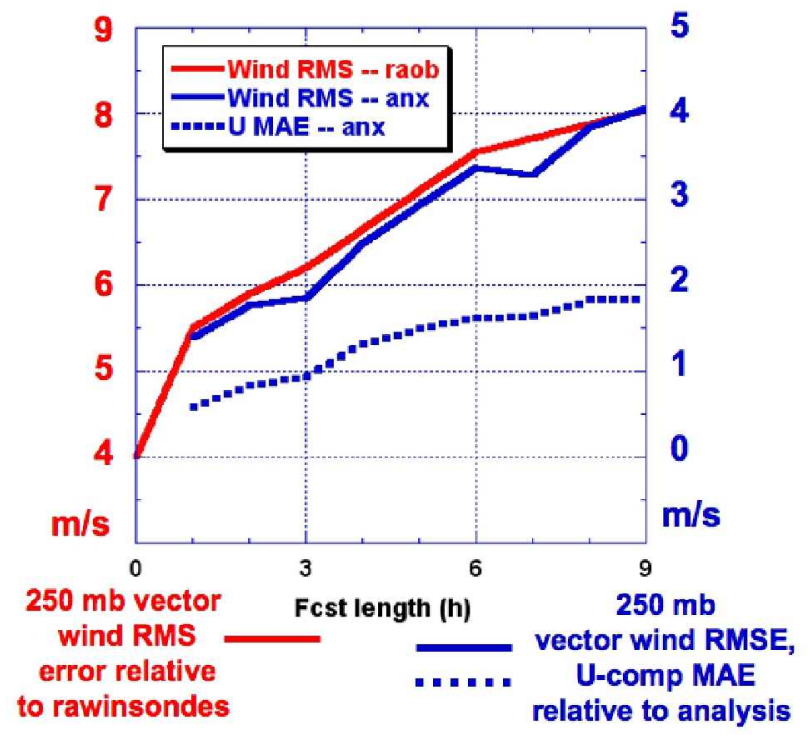

Figure 4: $250 \mathrm{mb}$ (33984 feet) Vector RMS Error and MAE

American Institute of Aeronautics and Astronautics 092407 


\section{Wind forecast uncertainty and spread-Skill relationship}

Having established that on average RUC upper-level wind forecast errors decrease with decreasing lead time and that a correspondence exists between forecast errors relative to the observations and to the analyses, we now examine uncertainty of the forecast wind fields as measured by a time-lagged forecast ensemble obtained from successive RUC model runs. Using forecast ensembles to generate uncertainty information is a common practice in numerical weather prediction, with much work focused on methods for generating optimal sets of ensembles. ${ }^{17,18}$ Traditional approaches have examined various methods for perturbing the model initial fields, model physics, boundary condition information, and even the use of multi-model ensembles. These approaches, which often involve running sets of 10-20 perturbed model runs are extremely computationally intensive and time consuming, and usually necessitate running the ensemble system at a degraded spatial resolution. Because the RUC is run every hour, it is possible to use older RUC forecasts valid at the same time to provide additional ensemble members. An obvious advantage is the great saving in computational time and resources as these runs are already available. As noted earlier, disadvantages are that the ensembles are not all equally likely and the ensembles may exhibit a large degree of correlation with each other. In this subsection, we examine the spread of a test set of RUC ensembles and evaluate its relationship to the Skill of the RUC forecasts.

As a proof of concept, the Skill of the RUC U component uncertainty field (calculated as the standard deviation of the $U$ component among the ensemble members and shown in Fig. 2a) at estimating the errors of the RUC wind forecasts valid 0000 UTC 3 April 2009 is evaluated. Again, in order to simplify the evaluation of Along-Track differences for the hypothetical flights described later, this portion of the study is restricted to considering only the U-component (east-west) of the RUC wind field and a single level ( $250 \mathrm{mb}$ or $\sim$ FL340) corresponding to en route flights. As shown in Fig. 2, strong spatial variability in the $U$ component uncertainty field (representing spread among the ensemble members) exists, which results in large variations in the wind forecast uncertainty along the hypothetical flight trajectories (superimposed on the figure). This can be illustrated by examining the two flight tracks indicated by the black arrows, Flight $\mathrm{C}$ which traverses from Minnesota into North Dakota and Flight B which traverses from South Carolina into Southern Indiana. Flight $C$ traverses a region of generally low uncertainty (indicated by the small values for standard deviation of the U-component of the wind among for the six ensemble forecasts) but the spread increases during the later portion of the flight track. In contrast, Flight B traverses a region of higher forecast spread, and the flight track is punctuated by two local maxima in spread, one during the middle of the flight and one at the end of the flight track. The effect of how these variations in spread in the wind field translate into variations in spread of an ensemble of predicted flight tracks is discussed later in the paper.

The variation in the spread (standard deviation from the six ensemble members), shown in Fig. 2, can be compared with the forecast - 0-hr Analysis forecast error for each member, which is shown in Fig. 5a-f (with the hypothetical flight tracks also indicated). The actual wind field at this time was almost entirely from west to east, so by examining a given flight track on each of the panels in Fig. 5 , the deviation in the flight time to be expected due solely to errors in the forecast wind field may be quantified. Thus for planes flying from east to west (the assumed direction in the hypothetical flight tracks), positive U-component errors imply forecast westerlies greater than analyzed westerlies, or a forecast of too strong a headwind. This would imply that an aircraft trajectory based on that forecast wind field would be too fast. Thus Flight $\mathrm{C}$ shows small errors, and a general trend of decreasing positive errors as the flight track progresses. For the Flight B flight track, the wind errors for the individual forecasts are larger and have a pattern of positive errors early, followed by large negative errors, and then positive errors at the end. The large negative errors in the middle part of the flight increase significantly with forecast leadtime. 


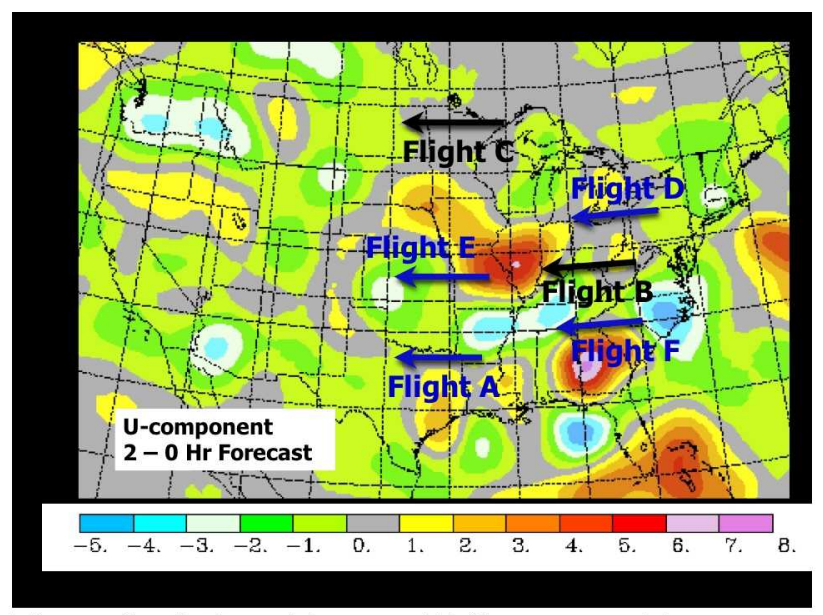

Figure 5a: 2-Hour Forecast U-Component Error

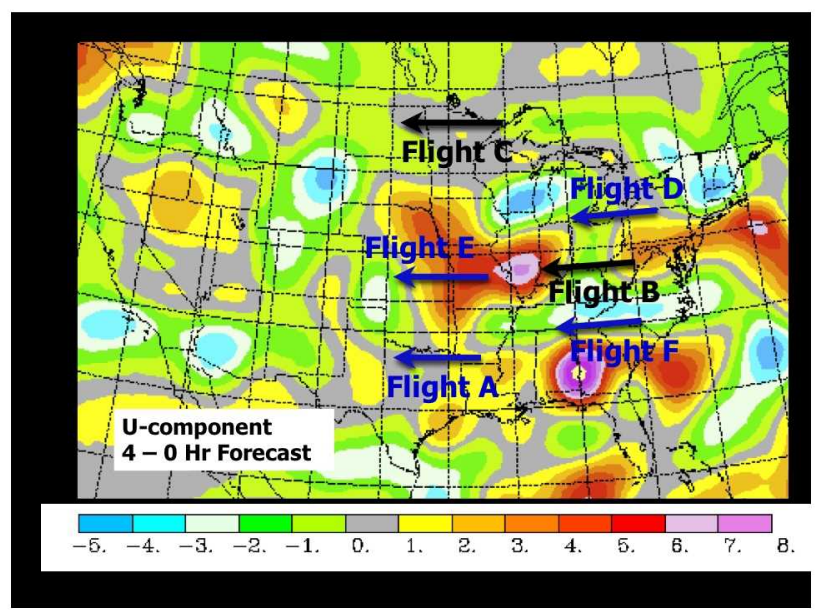

Figure 5c: 4-Hour Forecast U-Component Error

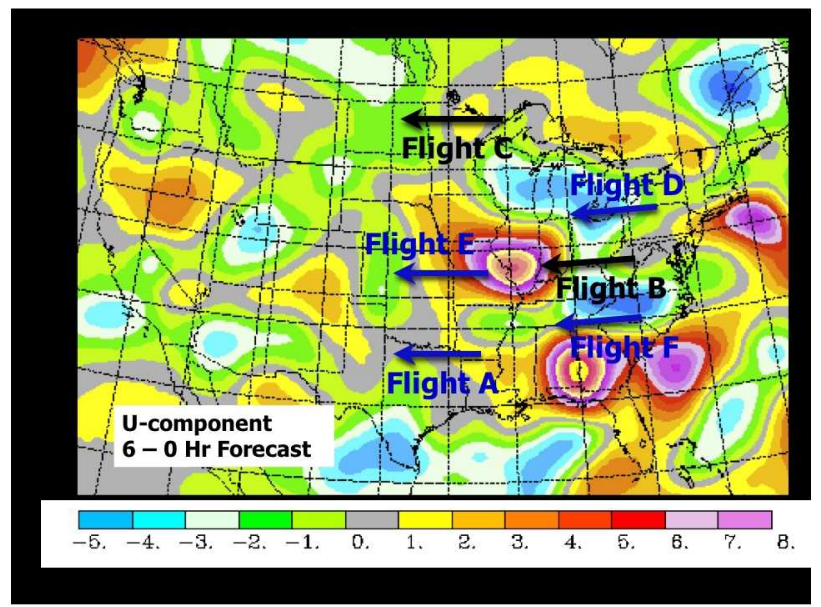

Figure 5e: 6-Hour Forecast U-Component Error

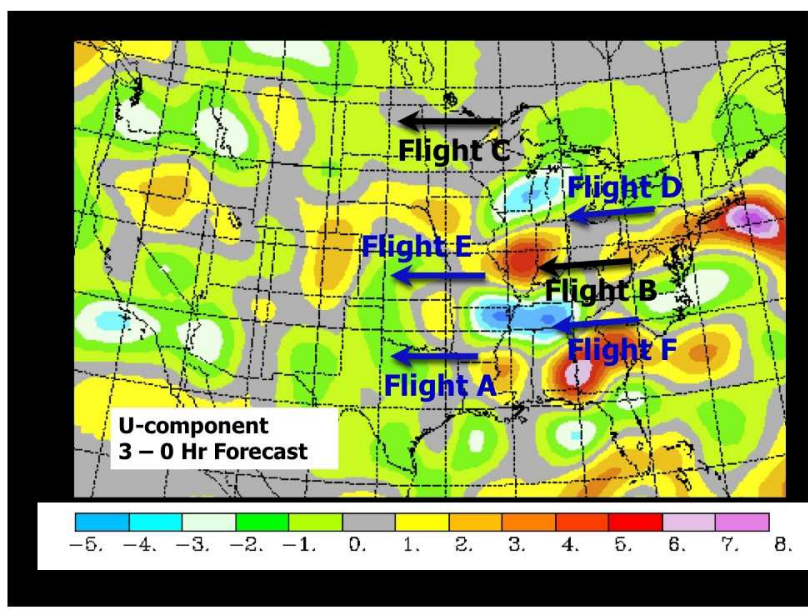

Figure 5b: 3-Hour Forecast U-Component Error

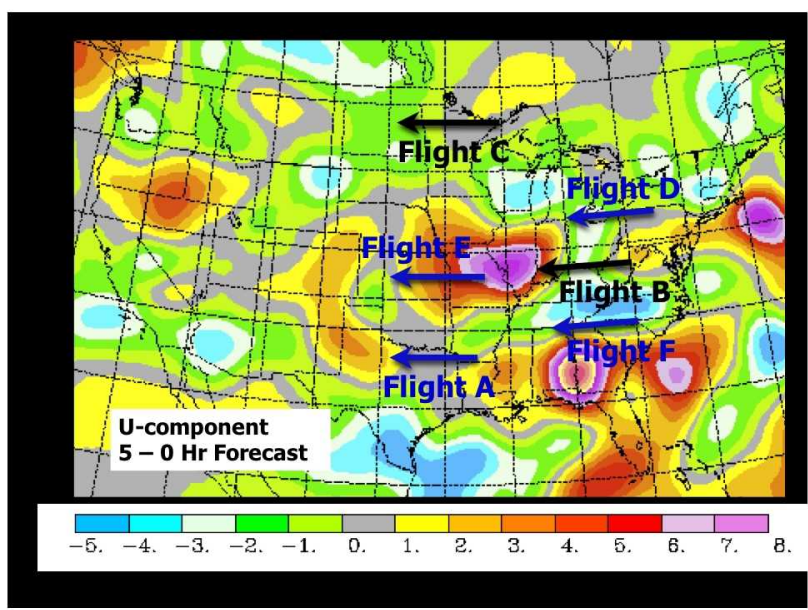

Figure 5d: 5-Hour Forecast U-Component Error

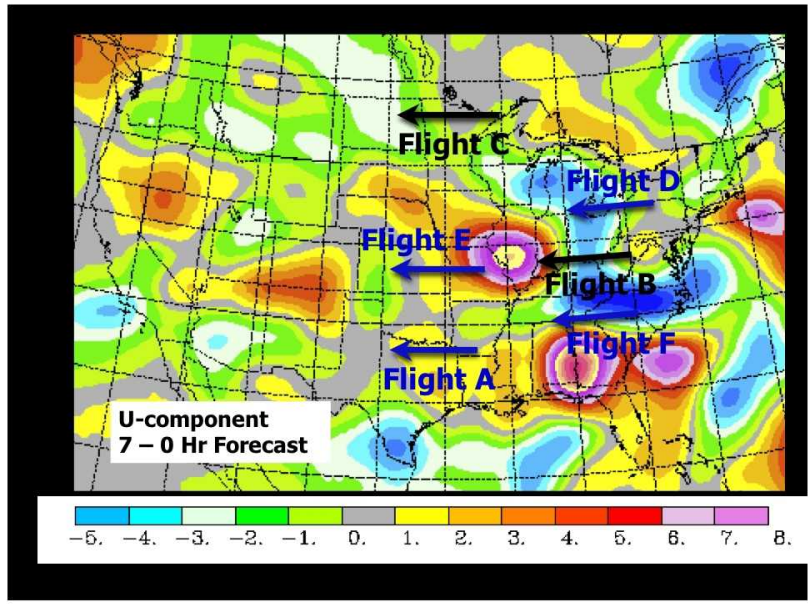

Figure 5f: 7-Hour Forecast U-Component Error

In order to quantify the ability of the time-lagged ensemble-based uncertainty field to predict the magnitude of the RUC forecast error, the linear correlation coefficient between the standard deviation of the forecast ensemble Ucomponent (shown in Fig. 2) and each of the mean absolute values of the U-component forecast error fields (shown in Fig. 5a-f) is analyzed. This result is plotted in Fig. 6 (along with the U-comp mean absolute error of each 
forecast). The correlation coefficient of the ensemble standard deviation with a given lead-time forecast ranges from about 0.35 for the 2-h forecast to near 0.75 for the 7 -h forecast. This relationship, in which the correlation between the spread of the ensemble and the Skill of the forecast increases as the forecast length increases, is as expected. The differences amongst the forecasts, which contribute to the ensemble spread, are precisely those differences that contribute to larger forecast errors as the forecast lead-time increases. This is evident in the similarity between the U-component MAE curve and the correlation curve. Thus, we see the inherent trade-off where, by using the freshest RUC model run to get the smallest forecast errors, some of the Skill in predicting the structure of those errors is sacrificed.

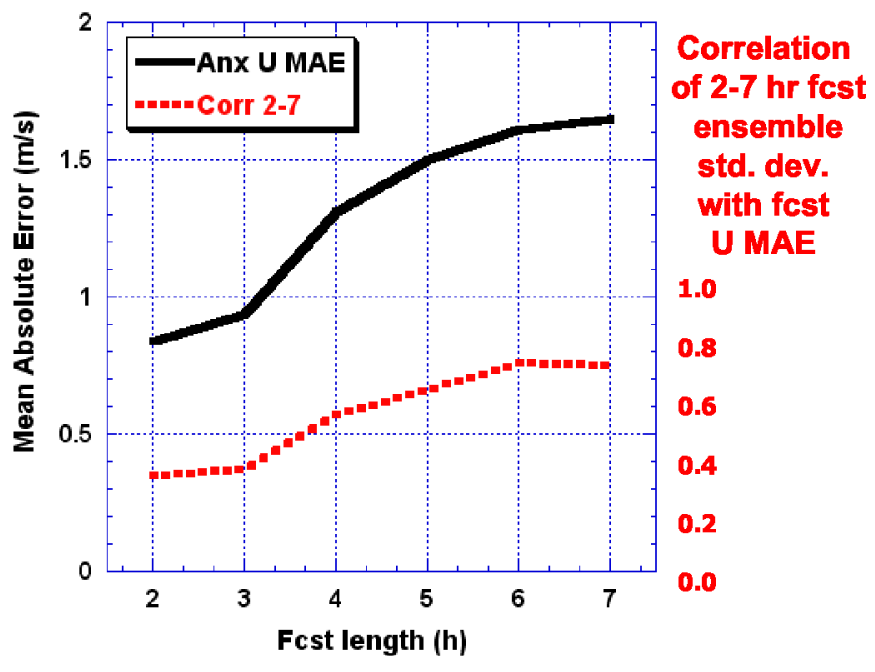

Figure 6: Comparison of Mean Absolute Error and Correlation of Ensemble Standard Deviation with Forecast U Component MAE

\section{B. Analysis of Along-Track Difference}

Cross track deviation was not considered in this study as the aircraft control behavior was modeled to constantly correct its course. Along-Track difference, however, was analyzed since aircraft trajectory predictions differing only by wind values along its heading would result in differences in ground speed. Along-Track difference is measured against a reference trajectory. The deviation is calculated by using the following equations and graphically shown in Fig 11:

$$
\begin{aligned}
& \Delta X=X_{r e f}-X_{\text {test }} \text { (4) } \\
& \Delta Y=Y_{\text {ref }}-Y_{\text {test }}(5) \\
& \text { Along-Track Difference }=\Delta X \sin \Psi_{r e f}+\Delta Y \cos \Psi_{r e f}
\end{aligned}
$$

Where $\Delta \mathrm{X}$ is the position difference of the reference trajectory $\mathrm{X}_{\text {ref }}$ and the test trajectory $\mathrm{X}_{\text {test }}$ and similarly for the Y-axis variables. This is used in conjunction with $\Psi_{\text {ref, }}$ the course of the reference trajectory to calculate the Along-Track Difference. 


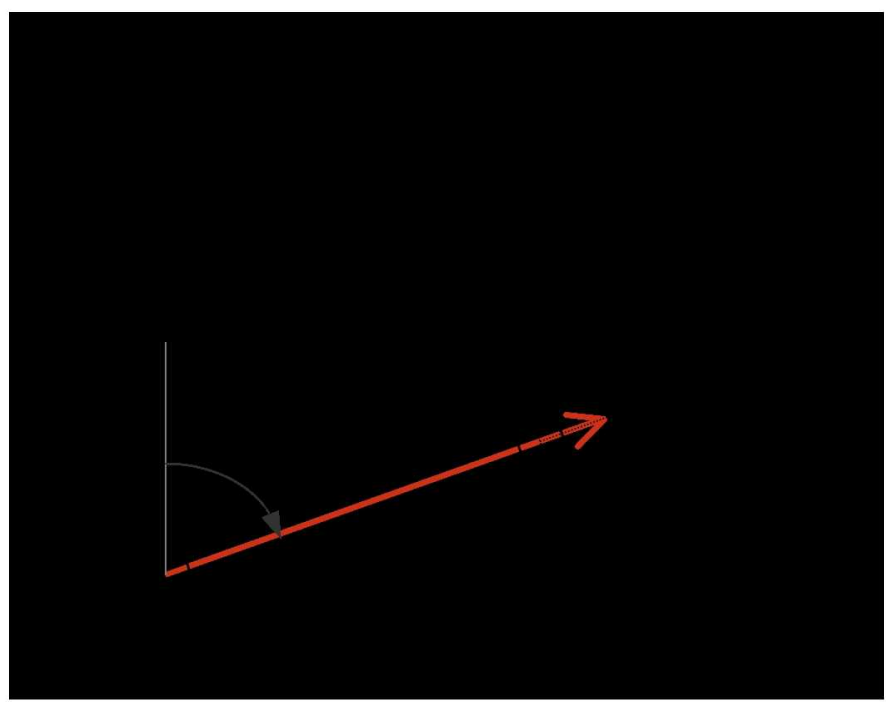

Figure 11. Along-Track Difference

For aircraft trajectory predictions with the same course, the Along-Track difference may be expressed with the following equation:

$$
\text { Total Along-Track Difference }=\sum_{i=1}^{n}\left[\left(V_{\text {ground_ref }_{-}}-V_{\text {ground_test }_{1}}\right) \times \Delta t\right]_{i}(7)
$$

Where $V_{\text {ground_ref }}$ and $V_{\text {ground_test }}$ are the ground speeds for the reference and test trajectories respectively. And $\Delta t$ is the time between each integration step, $i$.

Substitution of equation 1 into equation 7 yields:

$$
\text { Total Along-Track Difference }=\sum_{i=1}^{n}\left[\left(\left(V_{\text {TAS_ref }}+V_{\text {wind_ref }}\right)-\left(V_{\text {TAS }- \text { test }}+V_{\text {wind_test }}\right)\right) \times \Delta t\right]_{i}(8)
$$

Where $V_{\text {wind_ref }}$ and $V_{\text {wind_test }}$ are the wind components contributing along the aircraft's flight path for the reference and test trajectories respectively.

As mentioned previously, many decision support tools that model an aircraft in cruise use the initial wind speed at the aircraft location, and the initial ground speed, to calculate a TAS. The TAS is then used to calculate the intended airspeed in terms of a Mach or CAS. For two aircraft predictions using different wind forecasts but initialized with the same radar track information, the ground speed will be the same but the Mach and calibrated airspeeds may be different due to the difference of the forecasted wind value. These intended airspeeds are held constant in cruise by CTAS. As shown in equation 8, this disparity in cruise speeds is an additional factor for the Along-Track difference along with the differing winds along the two predicted flight paths. The difference in cruise speeds will always cause continual growth of the Along-Track difference over time whereas varying wind forecast uncertainty patterns may allow the Along-Track difference to converge over time.

If the intended airspeed is known (or is assumed) then the reference trajectory prediction and test trajectory prediction use the same airspeed. Assuming that the effect on airspeed due to other atmospheric properties such as temperature, density, and pressure are negligible, the only difference between the two trajectory predictions will be the influence due to wind. In this case, by reducing equation 8 to the following, the total Along-Track difference can be approximated: 


$$
\text { Total Along-Track Difference } \approx \sum_{i=1}^{n}\left[\left(V_{\text {wind_ref }}-V_{\text {wind_test }}\right) \times \Delta t\right]_{i}(9)
$$

Figure 12 shows an example of the relationship of the rate of Along-Track difference with respect to time and the difference of the $\mathrm{U}$ and $\mathrm{V}$ component wind speeds. In this case, the Along-Track difference is the trajectory generated using the 2-hour forecast referenced to the trajectory generated from the 0-hr Analysis forecast for an aircraft with a heading of 240 degrees. The $U$ and V component wind speed differences are from the 0 -hr Analysis forecast minus the 2-hour forecast projected along the aircraft's flight path. The aggregate influence of the wind speeds match the trend of the Along-Track difference growth rate described in Equation 9.

Figures 13 and 14 show the Along-Track difference as a function of time for Flights $\mathrm{B}$ and $\mathrm{C}$ respectively. The 0-hour Analysis forecast is used as a reference to show a comparison to a "truth" forecast. The accumulation of differences of the RUC wind fields along the aircraft flight track is shown. The Along-Track differences are calculated using the reference trajectory minus a test trajectory produced from a particular wind forecast (see Equation 9). A positive Along-Track difference, indicates that the trajectory generated from the reference forecast is ahead of the trajectory generated by the forecast.

The ensemble forecast

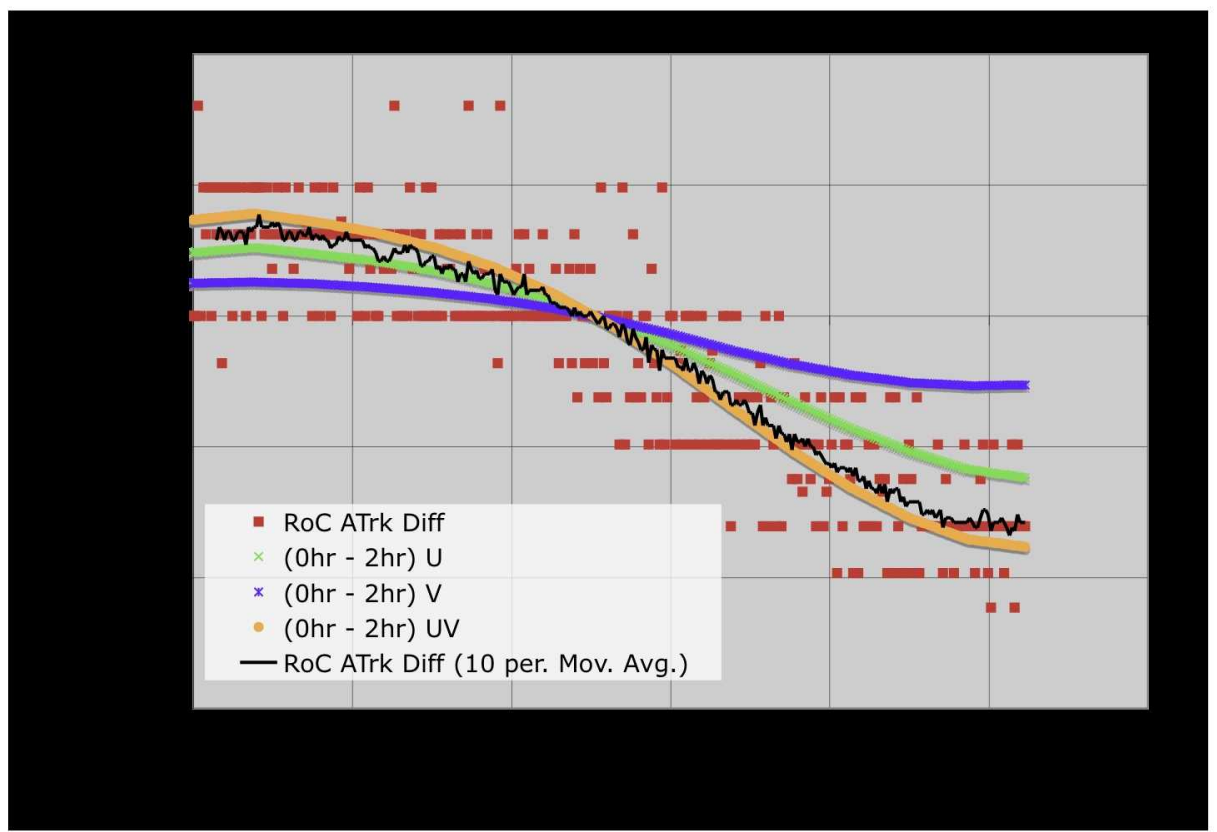

Figure 12. Along-Track Difference of Trajectories and Wind Differences from a 0-hr Analysis forecast and $2 \mathrm{hr}$ Forecast. Aggregate influence of the $U$ and $V$ component wind speed differences match the Along-Track difference growth rate.

average trajectory is generated from a wind forecast that is the mean average of the 2-hour through 7-hour forecasts. As mentioned previously, the first plot, Flight B (as shown in Figs 2a and 5), are from trajectory predictions where the aircraft flies through varying regions of low and high wind uncertainty. The second plot, Flight $\mathrm{C}$, is. from trajectory predictions in a region of low wind uncertainty. Overall Flight $B$ is flown within a region of higher wind uncertainty than Flight C. These uncertainty characteristics for the two flight paths are shown in Figure 2. Both flights are flown at a heading of 270 degrees to focus the investigation on a single axis of wind uncertainty.

The ensemble plus uncertainty and ensemble minus uncertainty Along-Track differences (light and dark grey lines respectively) use wind forecasts consisting of the standard deviation of the $U$ wind component added and subtracted (respectively) to the wind vectors of the ensemble forecast average. These two Along-Track differences outline one standard deviation of Along-Track difference over time for trajectories generated from the wind forecast members of the ensemble. The shaded region highlights this spread of possible Along-Track differences based on the accumulation of uncertainty along the flight track. This shows that the RUC uncertainty depicted in Fig. 2a translates into a time-dependent, growing trajectory prediction uncertainty. Flight B (Figure 13) illustrates a case where the trajectory from the 2-h RUC forecast provides a much better match to the 0 -hr Analysis forecast-based trajectory (the reference trajectory) than the trajectory from the ensemble mean. Overall, the large spread of the individual tracks from the various RUC ensemble members and the associated large spread of the shaded uncertainty cone (nearly $3.5 \mathrm{nmi}$ a $40 \mathrm{~min}$ ) is shown in Figs. 13. In contrast, the uncertainty for Flight $\mathrm{C}$ was much less as indicated in Fig. 14 by the Along-Track difference for the various tracks and the small spread of the shaded cone of uncertainty (around $1.5 \mathrm{nmi}$ at $40 \mathrm{~min}$.). 
As mentioned earlier in Section II, the predicted cruise speed used throughout the flight is typically derived from the wind and ground speed at the initial prediction point. Cruise speeds varying from $0.76,0.78$, and $0.80 \mathrm{Mach}$, amount to differences of approximately 10 knots TAS between each speed. Due to this difference in speed, an Along-Track variation between each trajectory would be approximately $8 \mathrm{nmi}$ at 40 minutes of elapsed time. This is significant when compared with the previous results of Along-Track variation due to flying through the wind field and highlights the importance of determining the correct intent speed of the aircraft.

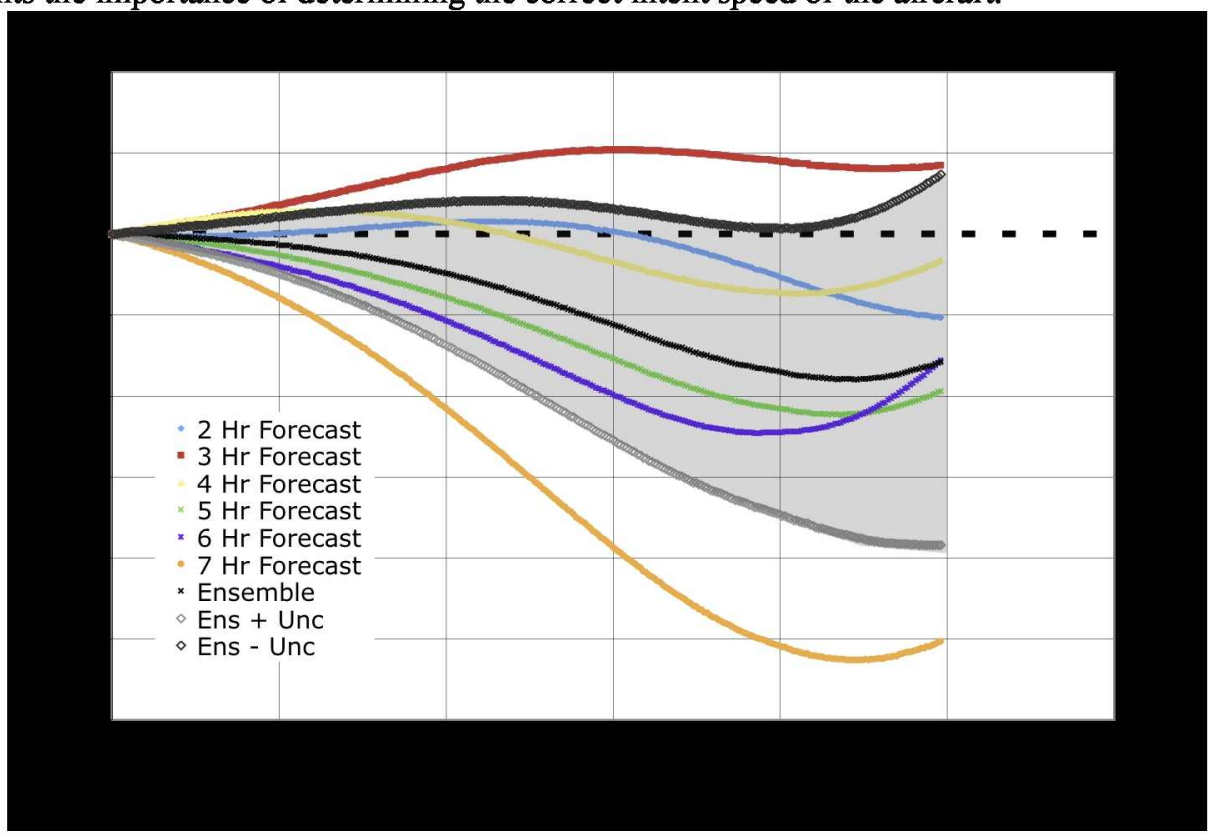

Figure 13. Flight B Along-Track Difference with 0-hr Analysis forecast as Reference

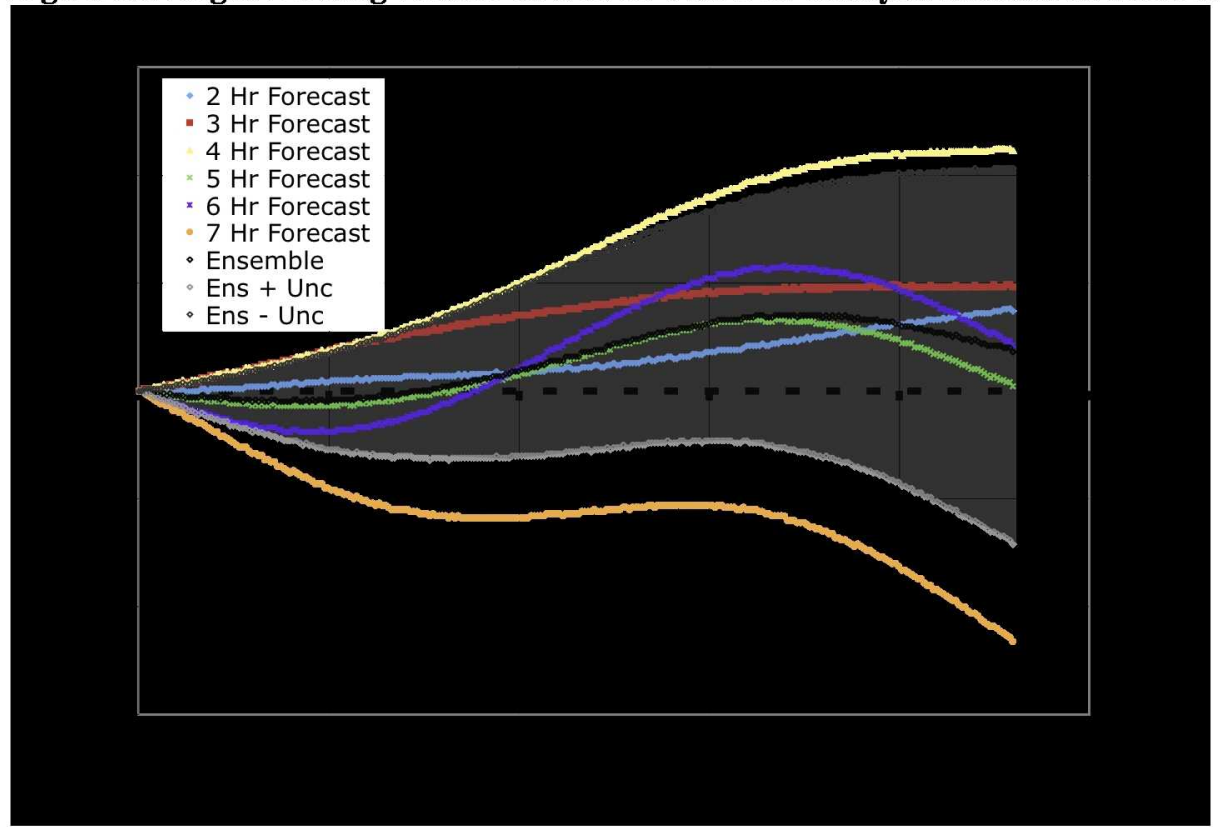

Figure 14. Flight C Along-Track Difference with 0-hr Analysis forecast as Reference

In some forecast cases such as the 5 -hour for Flight $\mathrm{C}$ (shown in Figure 14), the Along-Track difference virtually cancels out over time. Another observation is that the shorter lead-time forecasts do not always yield the most accurate trajectories, even though the shorter lead-time forecasts had smaller overall errors (domain-wide average) as shown in Fig. 4. These results emphasize the regional nature of the forecast errors (as can be seen in Fig. 5), and the need to capture regional variations in the uncertainty field. 
Using these plots, the Along-Track difference due to wind uncertainty at a particular time can be determined. The desired time is used to determine the difference between the ensemble mean forecast and the ensemble plus uncertainty" as well as the "ensemble minus uncertainty" forecasts. For the cases shown in figures 13 and 14, the uncertainty due to wind variability at 20 minutes is approximately less than 0.9 nautical miles for Flight B and 0.5 nautical miles for Flight $\mathrm{C}$.

Flight cases with non-cardinal headings were used to examine the interaction of the $\mathrm{U}$ and $\mathrm{V}$ component wind uncertainty. Flight B3, flown in the same region as Flight B is shown in Figs. 15 and 16. The flight path uses the same flight path mid-point as Flight B, but has a course of 240 degrees. Besides the standard forecasts $(2$ hour through 7 hour), the 0-hr Analysis forecast, and the ensemble average forecast, Flight B3 was flown with four additional wind maps. Each of these wind maps uses the ensemble average forecast with the $U$ and $V$ uncertainty values applied in the cardinal directions to apply the maximum influence of uncertainty. For a course of 240 degrees one wind map has the uncertainty applied in the $+\mathrm{U}+\mathrm{V}$ directions and the other has it applied in the $-\mathrm{U}$ and $-\mathrm{V}$ directions.

Figure 15 illustrates the Along-Track differences using the 0-hr Analysis forecast as the reference trajectory. The distribution of the trajectories of the 2-hour through 7-hour forecast is very similar to those of Flight B (see Figure 13) with the trajectories from the 3-hour and 7-hour forecasts again diverging the most. As in the previous, U-component only case, the shaded area depicts the cone of uncertainty, defined using the ensemble mean wind field with the plus and minus one standard deviation from the time-lagged ensemble. A sample of the uncertainty applied to the forecast used in an operational environment (2-hour) is shown in Figure 16. Comparing Figs 15 and 16 , we see that the 2-hour forecast is initially less accurate than that of the ensemble mean, but is more accurate after about $35 \mathrm{~min}$. On average the 2-hour forecast is more accurate but the ensemble mean is more robust to occasional regions of very large errors.

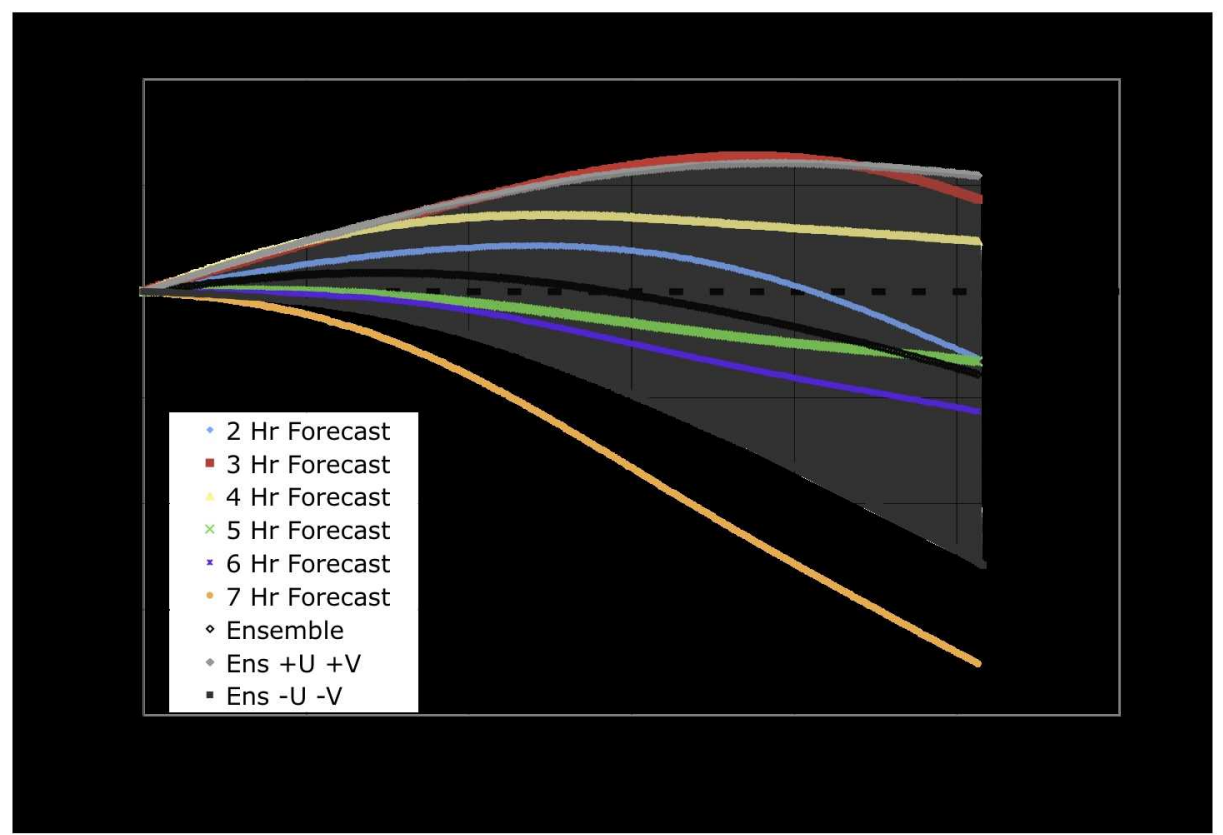

Figure 15: Along-Track Difference of Flight B3 with the 0-hr Analysis forecast as the Reference 


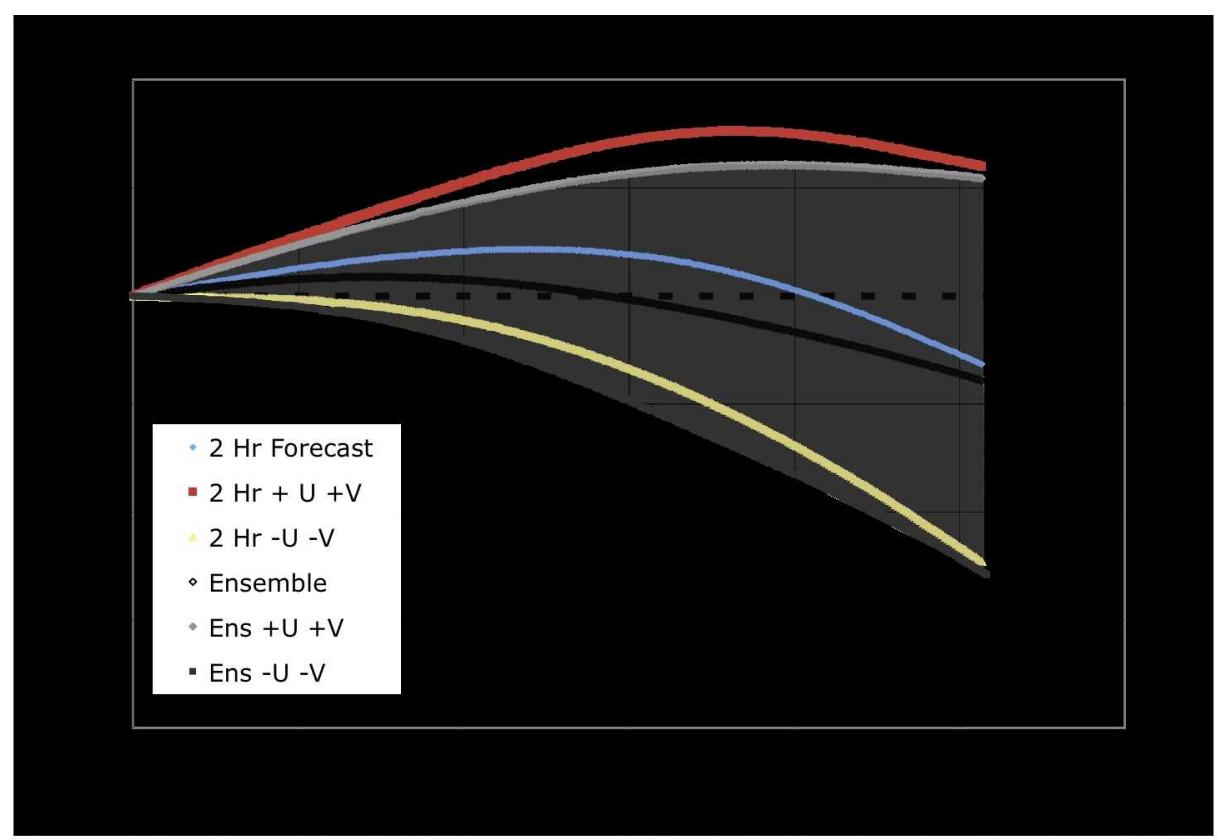

Figure 16: Along-Track Difference of Flight B3 Showing Application of Win Uncertainty to 2-Hour Forecast with 0-hr Analysis forecast as the Reference

\section{Discussion}

This study used the standard deviation from a time lagged forecast ensemble to generate a wind uncertainty value. Other ensemble techniques such as the use of multi-model ensembles may also be used but the time lagged ensemble technique is simpler as the data for the ensemble members is readily available. Either an ensemble technique or a more sophisticated wind uncertainty model can be used. One possible method would be to combine the statistical model described by Mondoloni ${ }^{8}$ with the approach described in this paper. Regardless of the method used to determine the wind uncertainty, it should be independent of how the application of wind uncertainty on trajectory uncertainty is performed.

The hypothetical flights were flown through different regions of space and experienced distinct patterns of forecast errors and uncertainty distribution. From the small number of samples using the method in this study, the amount of trajectory uncertainty had a positive correlation with the amount of wind uncertainty along the flight paths. Forecasts with the shorter lead times had smaller overall errors (geographic domain-wide average), however using these forecasts for trajectory prediction were not always the most accurate (using the trajectory from the 0 -hr Analysis forecast as a proxy for "truth"). This highlights the regional nature of the forecast errors and the importance of capturing the regional variations in the wind uncertainty field.

This study used wind forecast files that had the wind uncertainty magnitudes applied in predefined directions. In an operational sense, however, the uncertainty data needs to be included with the RUC forecast as a separate data set from the wind forecast data. The trajectory predictor must be able to extract the wind uncertainty data and dynamically apply it relative to the heading of each individual aircraft. Other methods to use wind uncertainty for trajectory prediction in an operational environment need to be explored. One possible method to determine the total Along-Track difference is to accumulate the Along-Track difference uncertainty due to the wind uncertainty that the aircraft encounters along its flight path. Computational load must also be considered in an operational environment as the number of additional calculations can put a strain on the DST.

The Along-Track difference due to forecast wind uncertainty can also be used to influence ATM metrics such as conflict avoidance buffer size. Obviously a buffer size that is too small will compromise safety, while an overly conservative size will limit the amount of traffic that can fly through congested airspace. The size of the $8 \mathrm{nmi}$ aircraft separation buffer used in CTAS consists of the $5 \mathrm{nmi}$ legal separation as well as an additional $3 \mathrm{nmi}$ which accounts for all sources of uncertainty. For ATM, a 20-minute look-ahead time is used for a typical conflict probe. By determining the Along-Track difference from the one standard deviation wind forecast at 20 minutes elapsed time (described in a previous section), the amount of separation buffer due to wind alone with a one sigma (68.2\%) 
confidence value can be found and added to the $5 \mathrm{nmi}$ legal separation. The trajectory uncertainty distance due to wind would be applied in the aircraft's Along-Track direction (Fig 17). The amount of separation buffer due to wind uncertainty can vary widely in different regions of space and is not a constant estimate. One possibility is to expand the separation buffer beyond $8 \mathrm{nmi}$ if the uncertainty due to wind exceeds the $3 \mathrm{nmi}$ margin used on top of the $5 \mathrm{nmi}$ legal separation. The additional amount would be the sum of the amount due to wind uncertainty plus a margin to account for other sources of uncertainty.

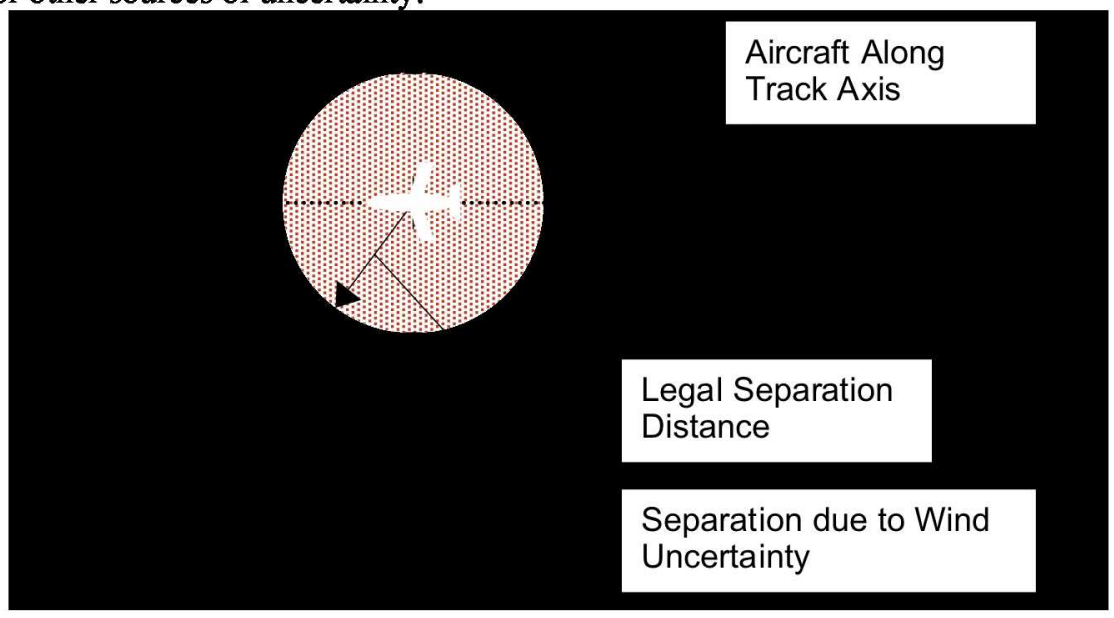

Figure 17: Separation Buffer due to Wind Uncertainty

Besides using the wind uncertainty for determining the size of the separation buffer, the wind uncertainty can also be used to aid with the time uncertainty associated with the arrival of an aircraft at a fixed point in space. Using the uncertainty value as an additional airspeed applied parallel to the aircraft's flight path, positively and negatively, an envelope for the of time of flight may be determined. As before with the Along-Track application, for a one standard deviation value, this would capture approximately $68.2 \%$ of the trajectories.

\section{Conclusion}

Errors in wind forecast data are one source of error in ATM predictions of aircraft trajectory location. This work describes a technique for estimating wind uncertainty and using it to estimate the level of uncertainty in hypothetical aircraft trajectory predictions. The technique uses a time-lagged ensemble of RUC model upper-level wind forecasts and estimates forecast wind uncertainty based on a statistical measure of forecast spread amongst the ensemble members. A key advantage of the time-lagged technique is that it identifies regional variations of uncertainty that are related to actual weather phenomena. This is confirmed by assessing the correlation between the wind uncertainty field and the wind forecast errors field.

The impact of the wind forecast uncertainty on trajectory prediction uncertainty was also examined. These test cases were very simplified using constant course headings and a snapshot of the wind forecast which is held constant for the duration of the forecast's hour of validity. Trajectory predictions were flown through different weather patterns and illustrated the distribution of wind uncertainty and its effect on the trajectory prediction. From these test cases methods were described which could be used to enhance ATM metrics such as separation assurance buffers and scheduling.

This proof of concept study demonstrates a method to use the wind uncertainty data in the en route flight regime. Future studies will address other subjects such as trajectories with altitude changes, multiple output time data from the RUC, probability density functions, and investigate different methods to apply the wind uncertainty data.

Wind uncertainty is but one element that contributes to the total uncertainty of a trajectory prediction. Accounting for other sources of uncertainty, such as aircraft performance modeling, observation, intent, and navigation, is also necessary to obtain the best estimates of aircraft trajectory uncertainty. A conglomerate model of uncertainty that includes all significant sources of uncertainty would result in a more complete analysis of trajectory prediction uncertainty against real aircraft trajectory data. 


\section{Acknowledgments}

From NASA Ames Research Center, the authors would like to acknowledge William Chan for his inspiration of this topic. Additionally the authors would like to thank him, along with Karen Cate and Steven Green for providing technical guidance.

Lastly the authors would like to thank David A. Darling of the University Affiliated Research Center who was essential for providing data processing support.

\section{References}

${ }^{1}$ Joint Planning and Development Office, "Concept of Operations for the Next Generation Air Transportation System, Version 2.0" Jun. 13, 2007.

${ }^{2}$ Schwartz, B.E., S.G. Benjamin, S.M. Green, and M.R. Jardin, 2000: Accuracy of RUC-1 and RUC-2 Wind and Aircraft Trajectory Forecasts by Comparison with ACARS Observations. Wea. Forecasting, 15, 313-326.

${ }^{3}$ R. Cole, S. Green, M. Jardin, "Improving RUC-1 Wind Estimates by Incorporating Near-Real-Time Aircraft Reports" Weather and Forecasting, Vol. 15, 447-460.

${ }^{4}$ Cole, R. E., Richard, C. M., Kim, S. K., Bailey, D. B., An Assessment of the $60 \mathrm{~km}$ Rapid Update Cycle (RUC) with Near Real-Time Aircraft Reports, Project Report NASA/A-1, MIT Lincoln Laboratory, Lexington, MA, 1998.

${ }^{5}$ R. Cole, S. Green, M. Jardin, B. Schwartz, and S. Benjamin, 2000: Wind Prediction Accuracy for Air Traffic Management Decision Support Tools. 3rd USA/Europe Air Traffic Management R\&D Seminar.

${ }^{6}$ Cole, R. E., Kim. S. K., A Study of Time-to-Fly Estimates for RUC and ITWS Winds, Ninth Conference on Aviation Range, and Aerospace Meteorology (ARAM), Orlando, FL, Amer. Meteor. Soc., 2000.

7 Thipphavong, D., "Analysis of Climb Trajectory Modeling for Separation Assurance Automation," American Institute of Aeronautics and Astronautics (AIAA) Guidance, Navigation, and Control (GNC) Conference, Honolulu, HI, 18-21 Aug. 2008.

8 Mondoloni , S. "A Multiple-Scale Model of Wind-Prediction Uncertainty and Application to Trajectory Prediction," 6th AIAA Aviation Technology, Integration and Operations Conference (ATIO), Wichita, Kansas, 25 27 September 2006

${ }^{9}$ Benjamin, S.G., G. A. Grell, J.M. Brown, and T.G. Smirnova, 2004a: Mesoscale weather prediction with the RUC hybrid isentropic-terrain-following coordinate model. /Mon. Wea. Rev. /*132*, 473-494

${ }^{10}$ Benjamin, S.G., D. Devenyi, S.S. Weygandt, K.J. Brundage, J.M. Brown, G.A.Grell, D. Kim, B.E.Schwartz, T.G. Smirnova, and T.L. Smith 2004b: An hourly assimilation-forecast cycle: The RUC. /Mon. Wea. Rev. /*132*, 495518.

${ }^{11}$ Green, S. M., and Vivona, R. A., "En route Descent Advisor Concept for Arrival Metering," AIAA-2001-4114, Guidance, Navigation, and Control Conference, Montreal, Canada, Aug. 2001.

${ }^{12}$ Swenson, H. N., Hoang, T., Engelland, S., Vincent, D., Sanders, T., Sanford, B., and Heere, K., "Design and Operational Evaluation of the Traffic Management Advisor at the Fort Worth Air Route Traffic Control Center," 1st USA/Europe Air Traffic Management R\&D Seminar, Saclay, France, June 1997.

${ }^{13}$ Schwartz, B.E., S.G. Benjamin, S.M. Green, and M.R. Jardin, 2000: Accuracy of RUC-1 and RUC-2 Wind and Aircraft Trajectory Forecasts by Comparison with ACARS Observations. Wea. Forecasting, 15, 313-326

${ }^{14}$ Benjamin, S. S., B. D. Jamison, W. R. Moninger, B. Schwartz, T. W. Schlatter 2008: Relative Forecast Impact from Aircraft, Profiler, Rawinsonde, VAS, GPS-PW, METAR, and Mesonet Observations for Hourly Assimilation in the RUC. 12th Conference on IOAS-AOLS, Amer. Meteor. Soc., New Orleans, LA

${ }^{15} \mathrm{Lu}$, C., H. Yuan, B.E. Schwartz, and S.G. Benjamin, 2007: Short-Range Numerical Weather Prediction Using Time-Lagged Ensembles. Wea. Forecasting, 22, 580-595.

${ }^{16}$ Coppenbarger, R. A., Lanier, R., Sweet, D., and Dorsky, S., "Design and Development of the En Route Descent Advisor (EDA) for Conflict-Free Arrival Metering," AIAA-2004-4875, AIAA Guidance, Navigation, and Control Conference, Providence, RI, 16-19 Aug. 2004.

${ }^{17}$ Slattery, R., and Zhao, Y., "Trajectory Synthesis for Air Traffic Automation," Journal of Guidance, Control and Dynamics, Vol. 20, No. 2, March/April 1997, pp. 232-238.

${ }^{18}$ Hou, D., E. Kalnay, and K. K. Droegemeier, 2001: Objective verification of the SAMEX'98 ensemble forecasts. Mon. Wea. Rev., 129, 73-91.

${ }^{19}$ Du, J., J. McQueen, G. DiMego, T. Black, H, Juang, E. Rogers, B. Ferrier, B. Zhou, Z. Toth and M. S, Tracton, 2004: The NOAA/NWS/NCEP short-range ensemble forecast (SREF) system: evaluation of an initial condition vs 
multi-model physics ensemble approach. Preprints, 16th Conference on Numerical Weather Prediction, Seattle, Washington, Amer. Meteor. Soc. 


\section{Performance of Trajectory Models with Wind Uncertainty Alan Lee}

NASA Ames Research Center

Steve Weygandt

NOAA Forecast Systems Laboratory

Barry Schwartz

Scientific Research Group

and

James R. Murphy

NASA Ames Research Center

William N. Chan

NASA Ames Research Center 


\section{Motivation}

- Accurate aircraft trajectory predictions are essential to Air Traffic Management

- Conflict Detection and Resolution

- Scheduling

- Trajectory prediction has many sources of uncertainty

- Aircraft trajectory predictions do not always account for wind forecast uncertainty.

- Relevant Work

- Bill Arthur - MITRE "Analysis of En Route Flight Time Error Due to Wind Prediction Errors"

- Stephane Mondoloni - CSSI Inc. "A Multiple-Scale Model of WindPrediction Uncertainty and Application to Trajectory Prediction" 


\section{Objective}

- Use wind uncertainty data to estimate its influence on trajectory prediction uncertainty

- Wind uncertainty data from NOAA Rapid Update Cycle (RUC) forecasts

- Strength of using this approach

- Wind uncertainty data is correlated with physical phenomena related to specific forecast patterns

- Different geographic regions will have different distribution patterns of uncertainty 


\section{Outline}

- Truth (0-hr Analysis Forecast)

- Method

- How wind uncertainty data was applied to trajectories

- Use of trajectory data for Air Traffic Management 


\section{RUC Upper Level Wind Vector RMS Errors}

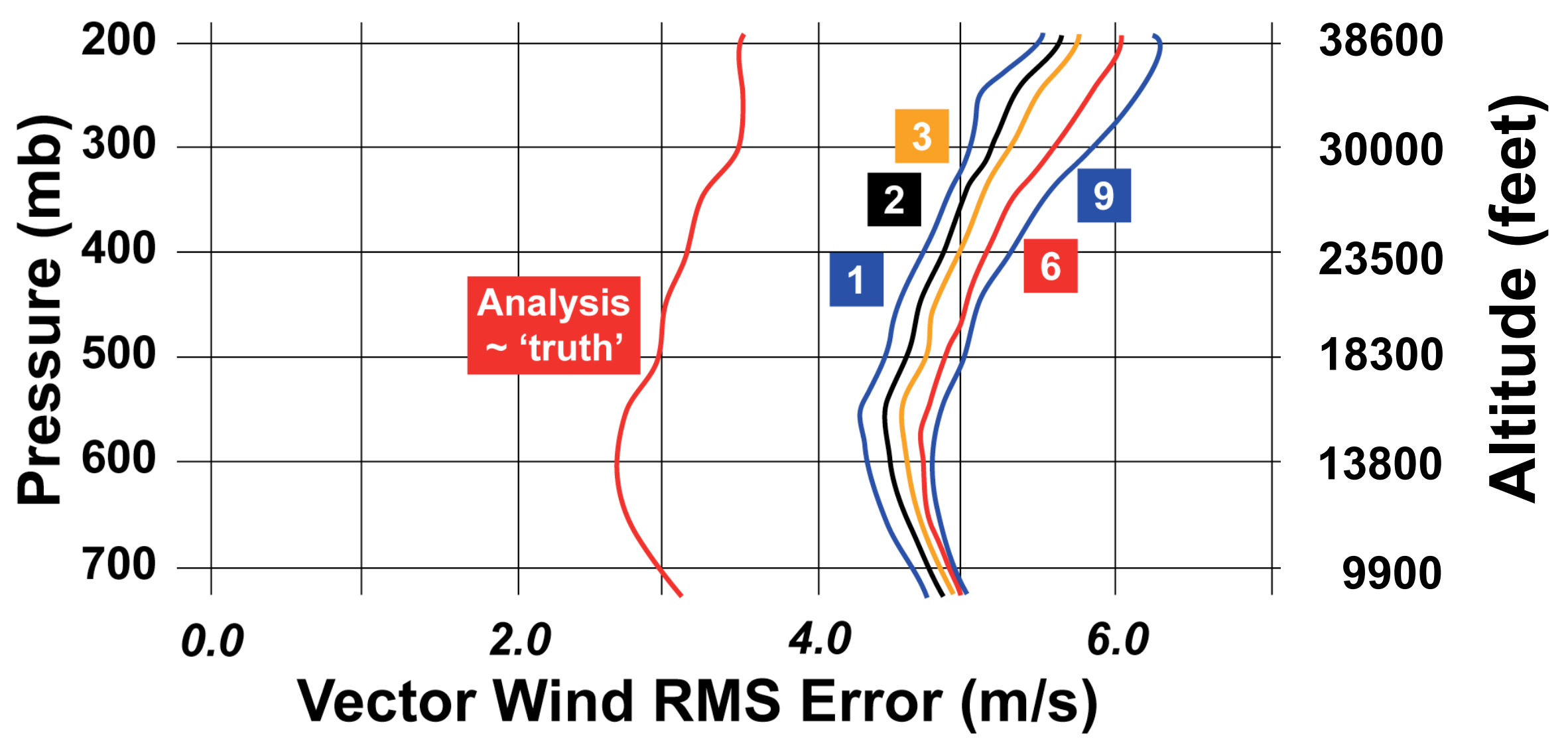




\section{Time-Lagged Ensemble Method}

- Method Used to Generate Wind Uncertainty

- Ensemble is composed of a set

- Actual forecasts of 2, 3, 4, 5, 6, 7-hour

- Valid for the same time

- Each ensemble member equally weighted

- Assumed a Gaussian distribution

- Average

- Standard Deviation (Uncertainty) 


\section{Application to Trajectory Prediction}

- Center TRACON Automated System (CTAS) used for trajectory generation

- Predefined wind maps with uncertainty

- $U$ and $V$ uncertainty applied in 4 combinations to a "base forecast"

- One standard deviation of uncertainty

- Hypothetical flights flown in a constant direction and altitude in different regions

- Single Axis

- U-Component uncertainty

- Non Cardinal A/C Heading

- U and V-Component uncertainty 


\section{Along-Track Difference Metric}

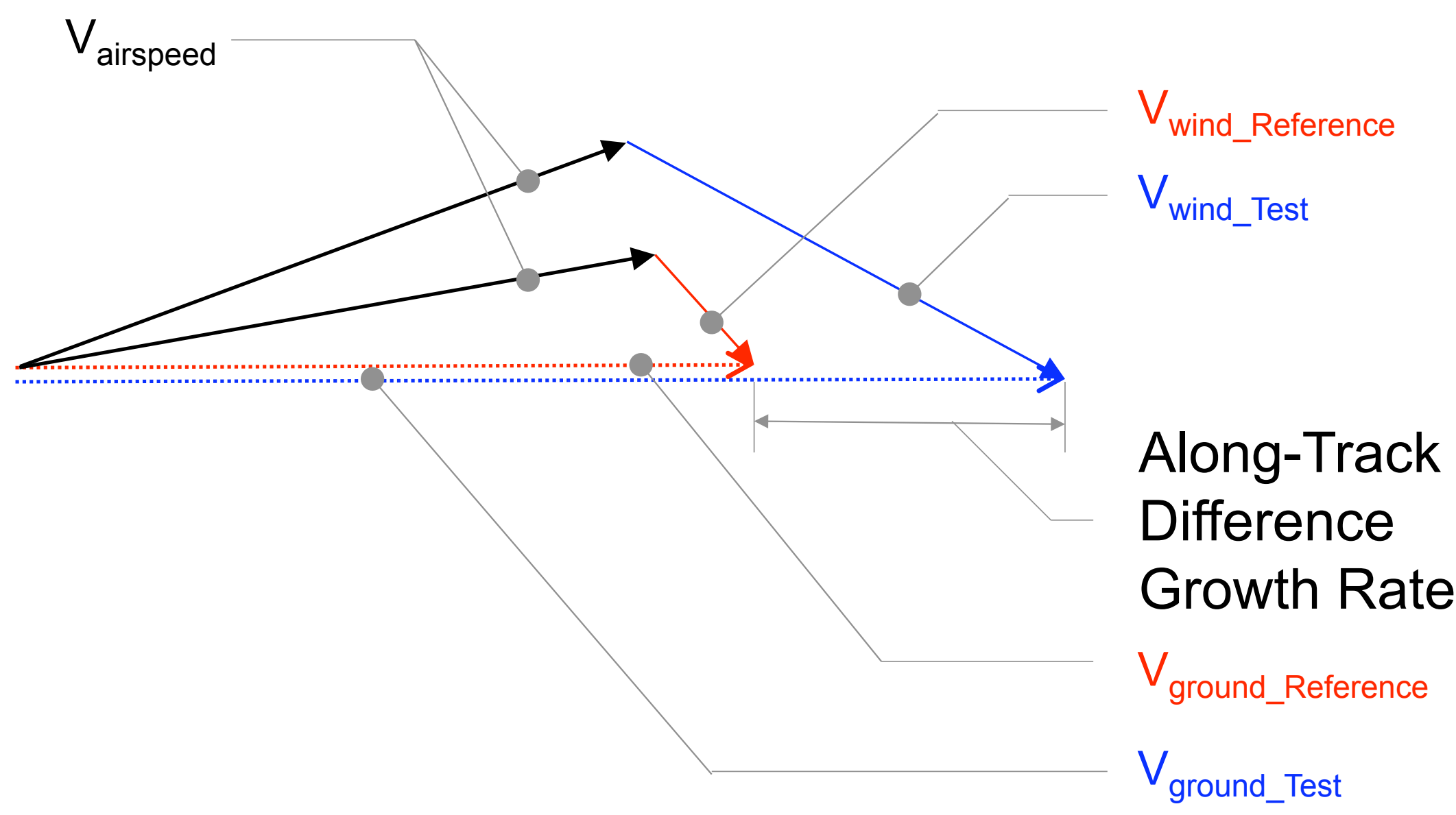




\section{Uncertainty Wind Map}

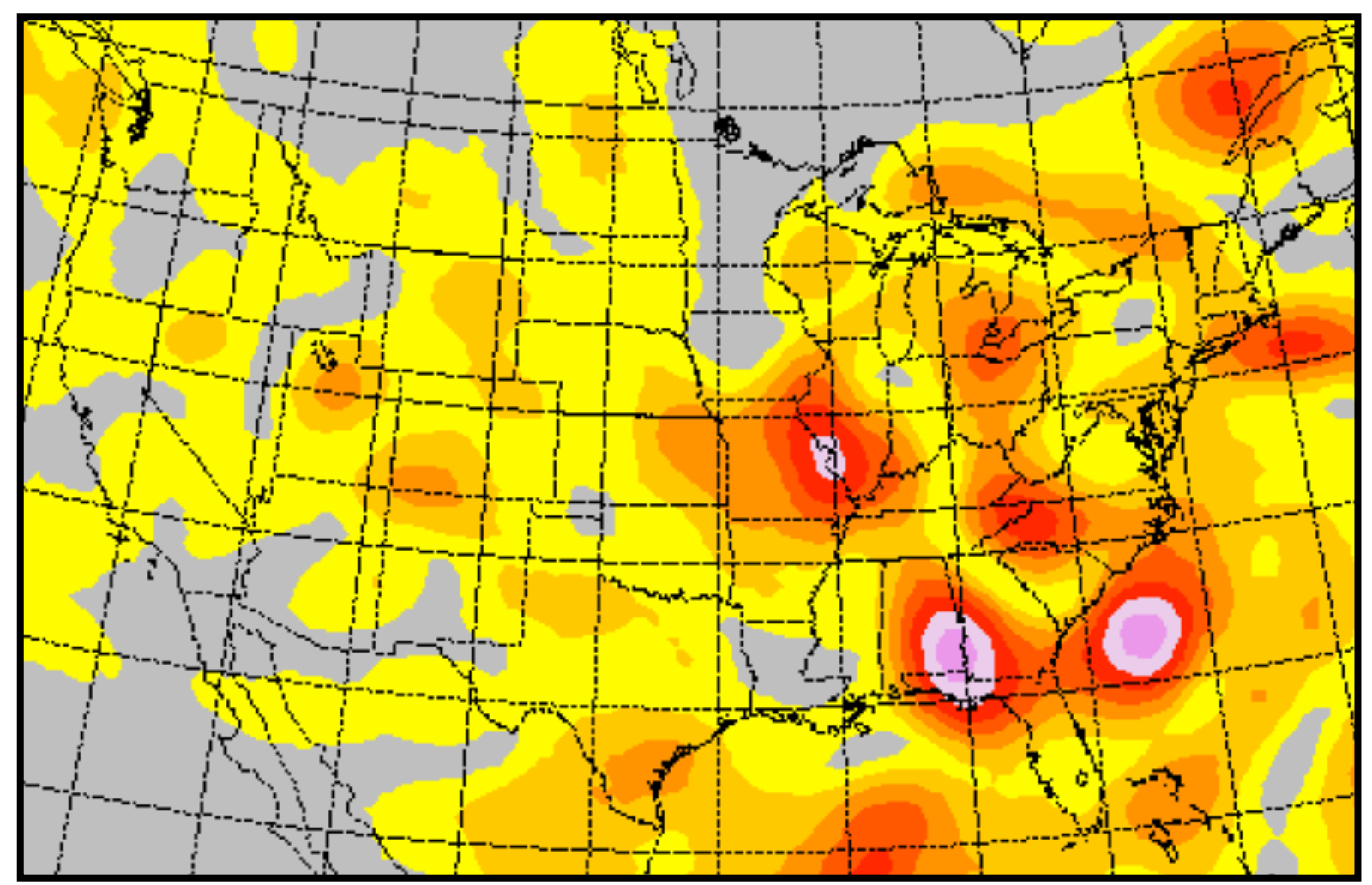




\section{Along Track Diff
Flight B}
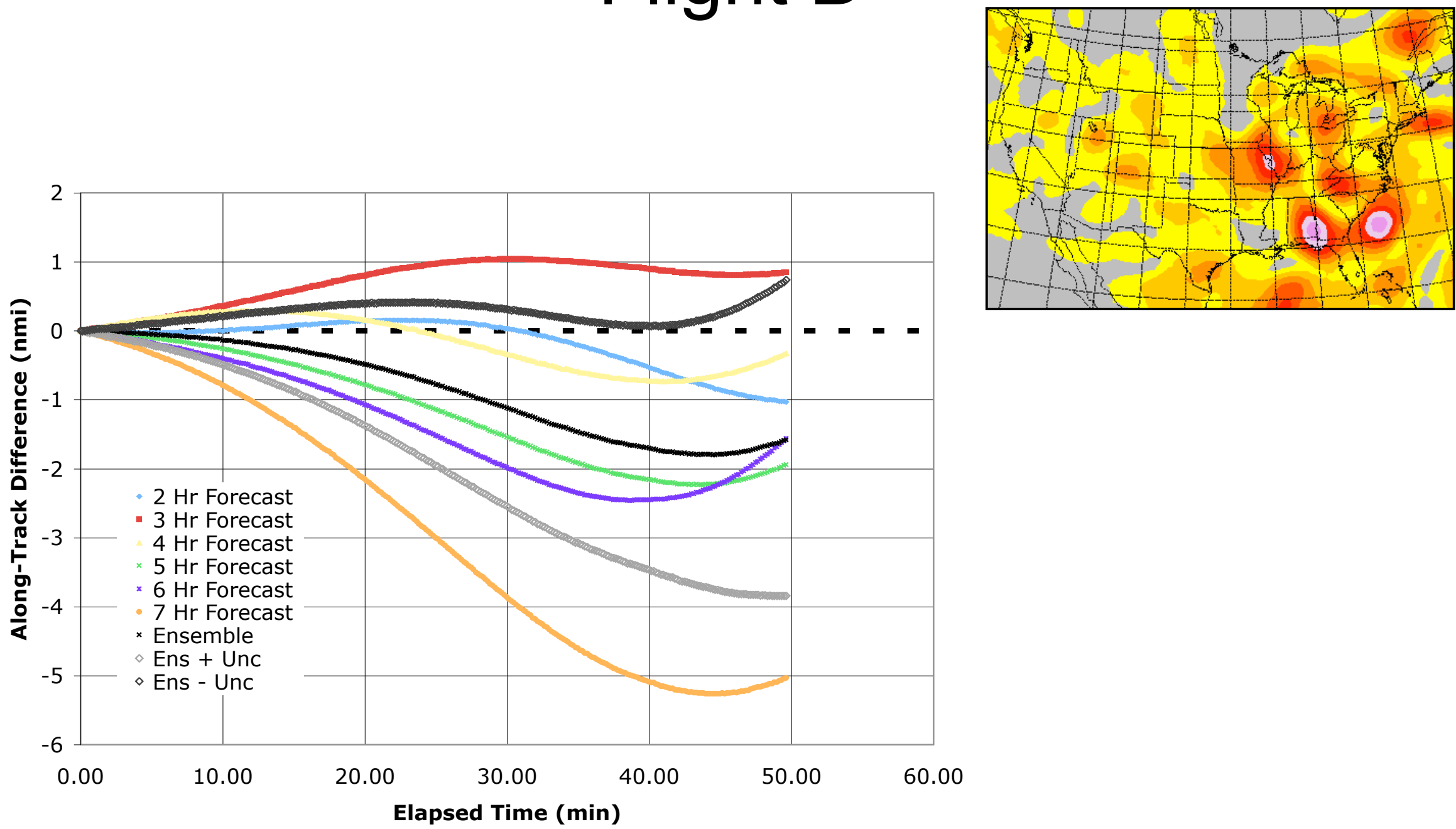


\section{Along Track Diffe
Flight C}
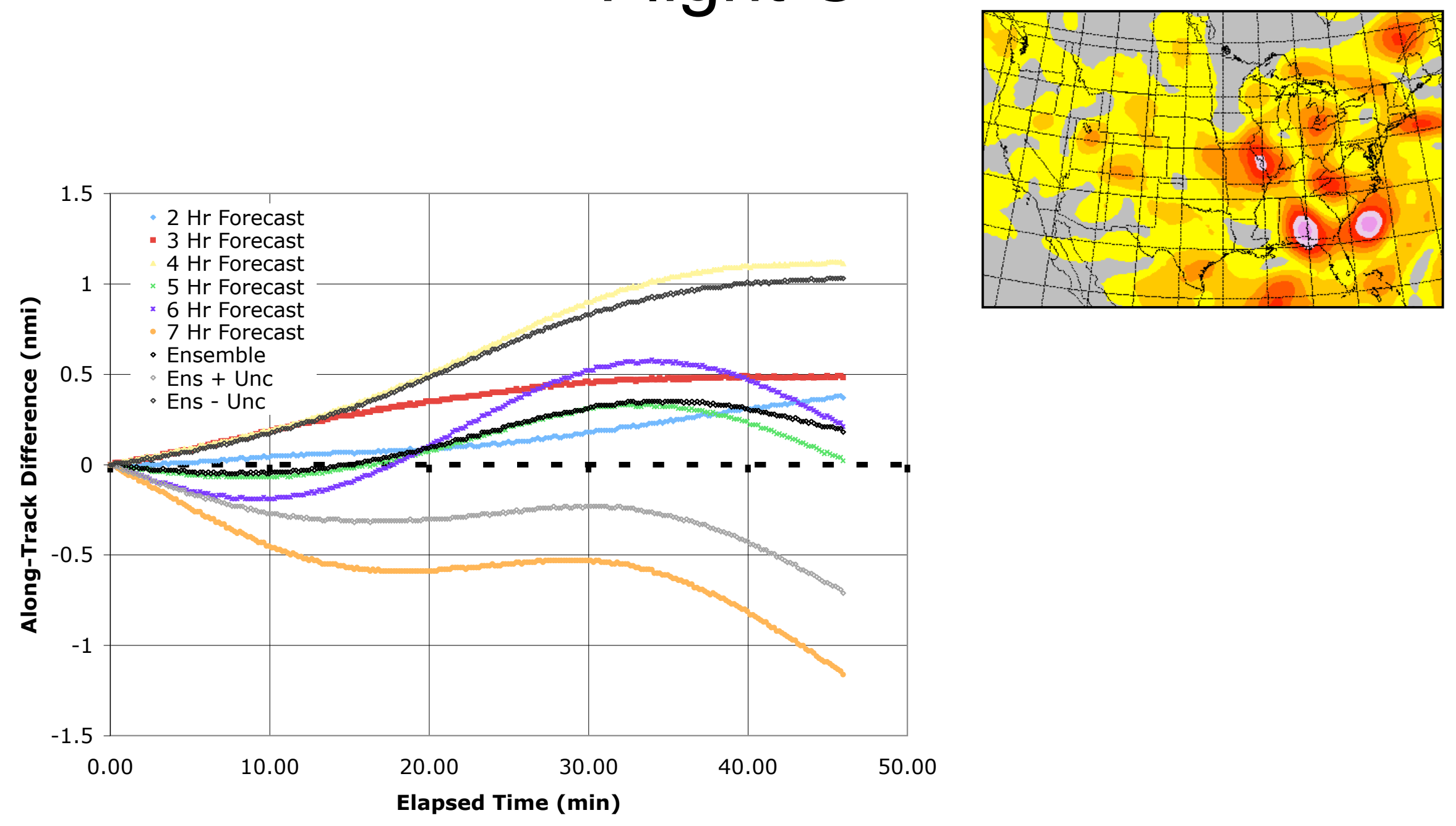


\section{Along Track Difference Continued}

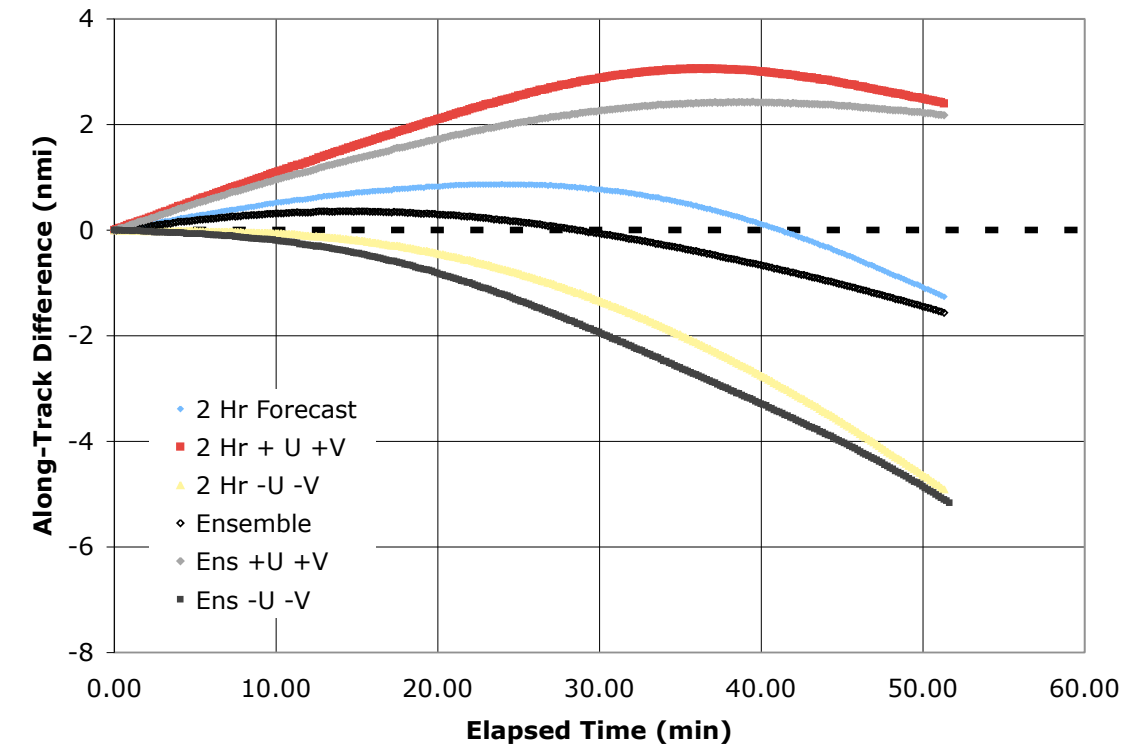

Uncertainty Applied to 2 Hour Forecast

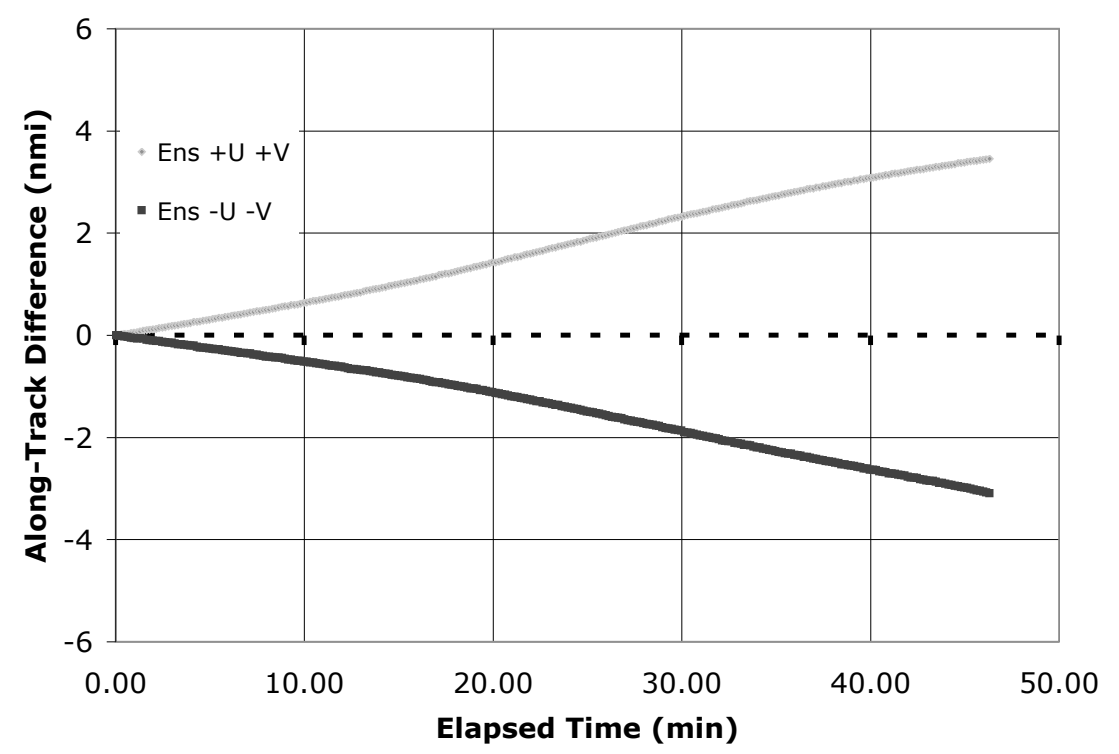

Ensemble Average as Reference Trajectory 


\section{Air Traffic Management Usage}

- Variable Size Conflict Separation Buffer 


\section{Future Work}

- More Data Samples

- Investigate use for climbs and descents

- Examine use of different probability distribution functions

- Explore different ways to use the uncertainty data

- Meteorological Validation of the Wind Uncertainty Model 


\section{Summary}

- Proof of concept study used wind uncertainty data to estimate its influence on trajectory prediction uncertainty.

- Model used wind uncertainty data derived from RUC forecasts

- Strength of this model

- Correlated with physical phenomena related to specific forecast patterns

- Captures different distribution patterns of uncertainty specific to the geographic region 


\section{Questions?}


BACKUP SLIDES 


\section{Comparison of Rawinsondes and Analysis Fit Forecast}

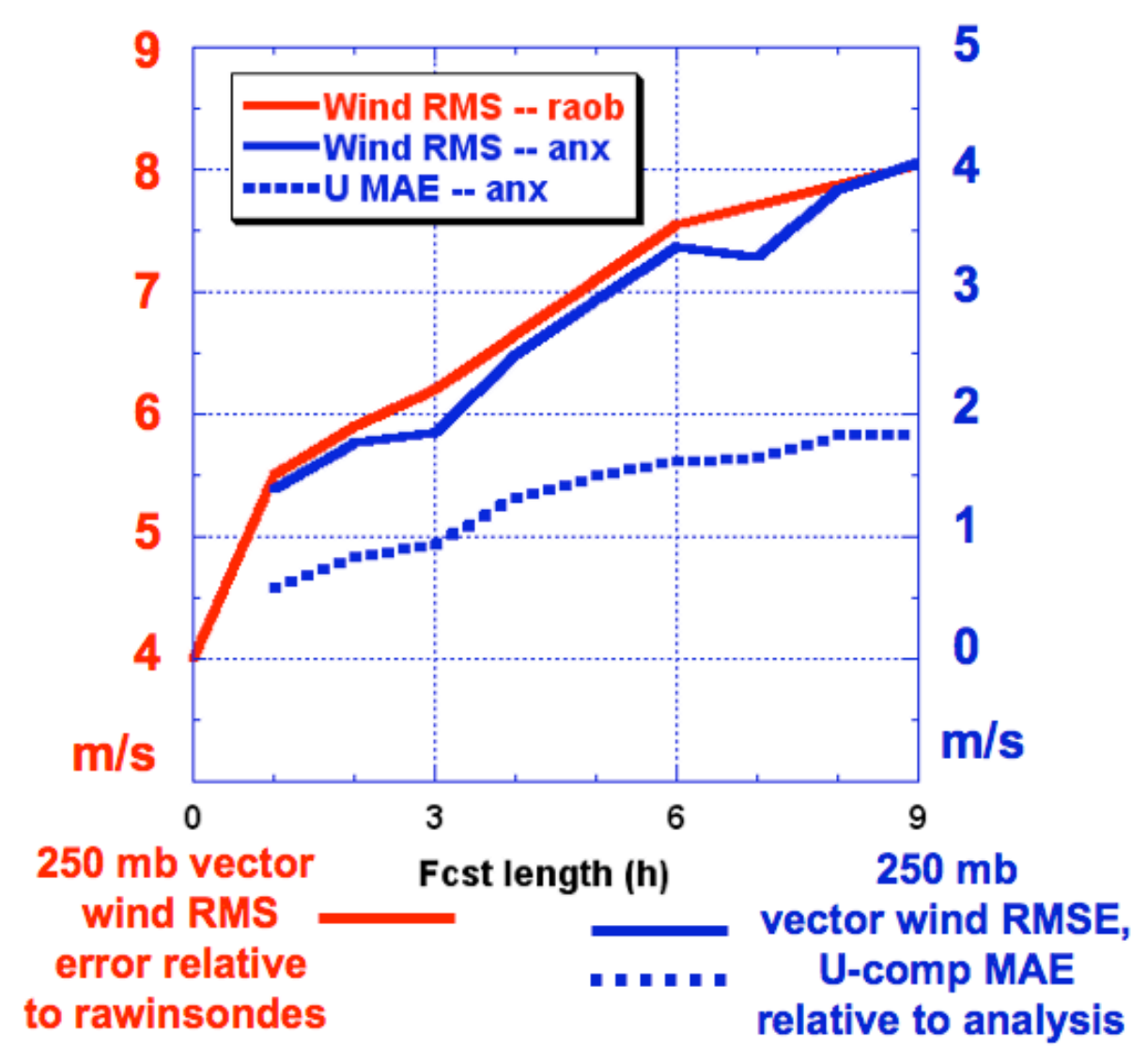




\section{Comparison of Mean Absolute Error and Correlation of Ensemble Standard Deviation with Forecast U Component MAE}

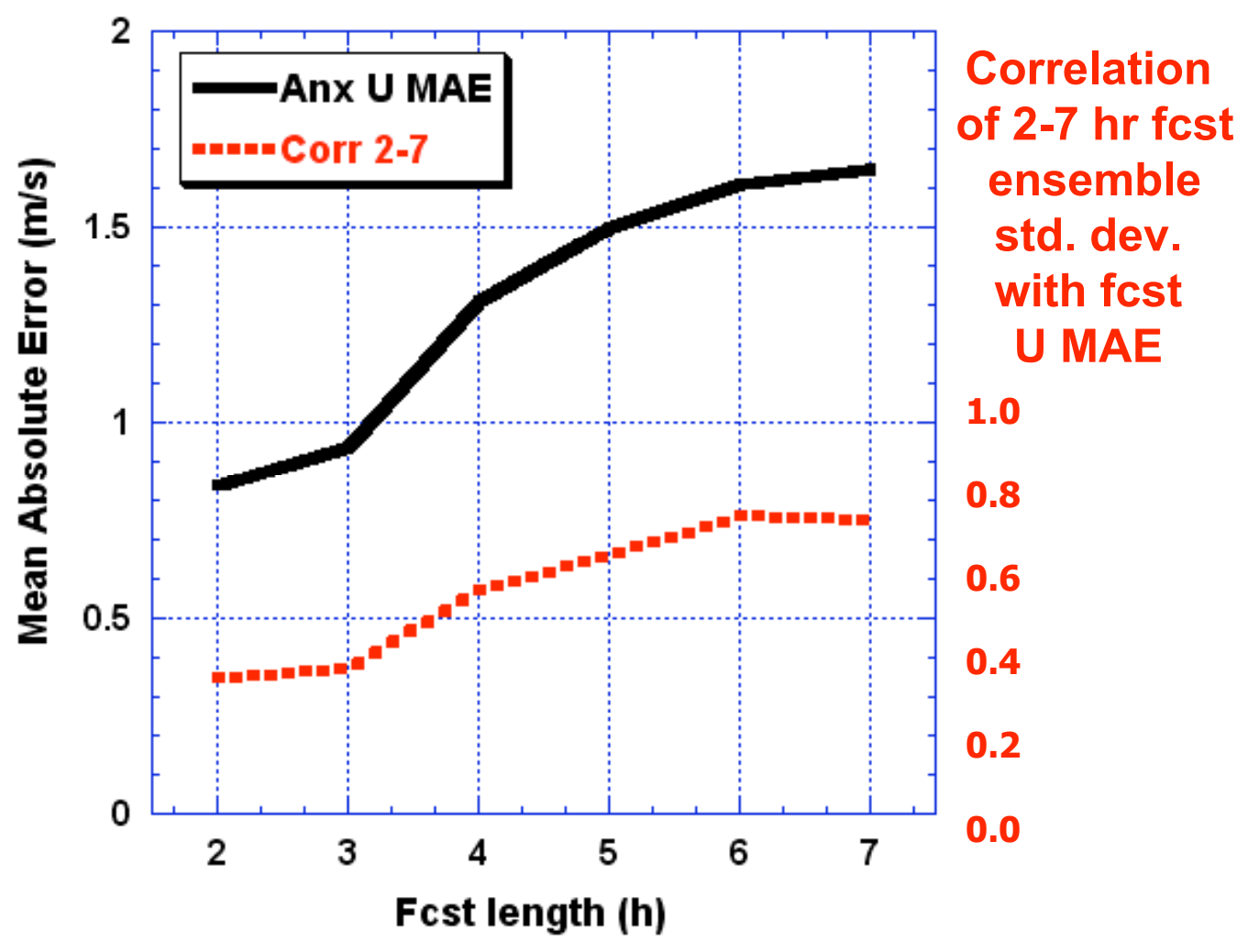




\section{2-Hr Forecast U-Component Error Map}

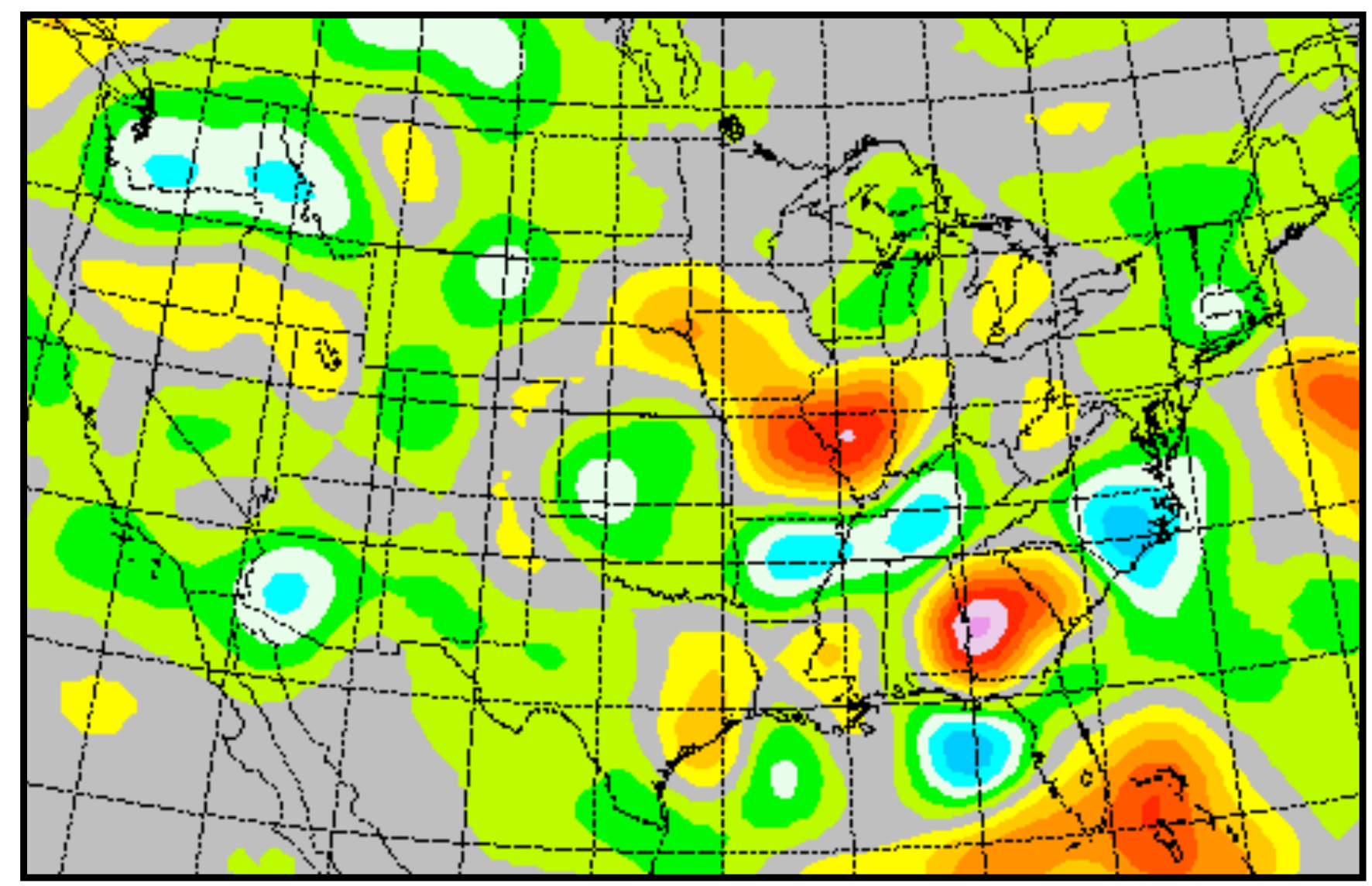




\section{Flight B - Heading 240 Along Track Difference}

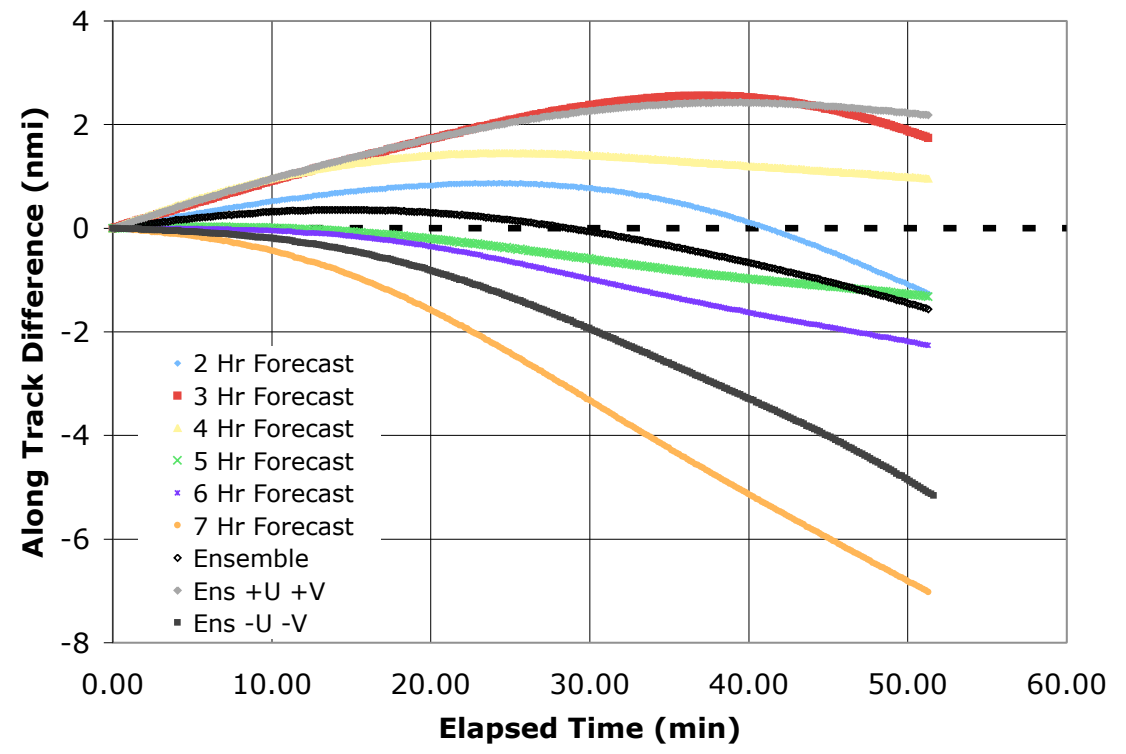

Flight B-Heading 240

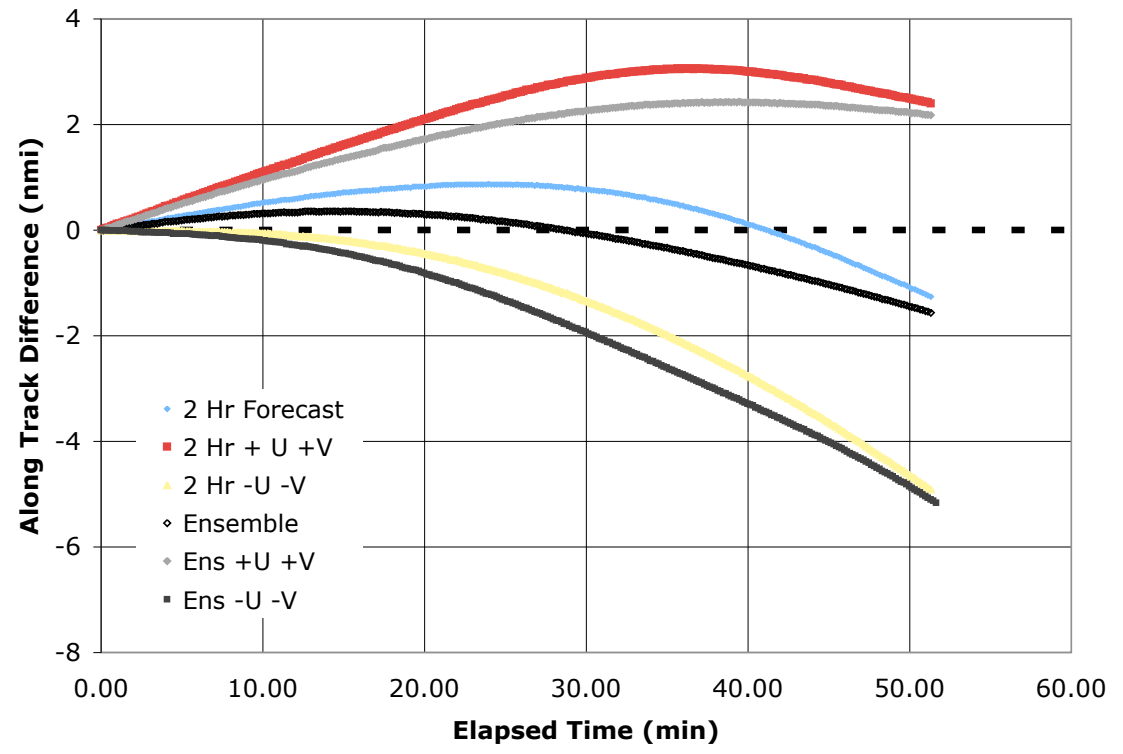

Uncertainty Applied to 2 Hour Forecast 


\section{Along Track Difference}

Along Track Difference

$$
\begin{aligned}
= & \Delta X \sin \Psi_{\text {ref }}+\Delta Y \cos \Psi_{\text {ref }} \\
= & \sum_{i=1}^{n}\left[\left(V_{\text {ground_ref }}-V_{\text {ground_test }}\right) \times \Delta t\right]_{i} \\
& \vec{V}_{\text {ground }}=\vec{V}_{T A S}+\vec{V}_{\text {wind }} \\
\approx & \sum_{i=1}^{n}\left[\left(V_{\text {wind_ref }}-V_{\text {wind_test }}\right) \times \Delta t\right]_{i}
\end{aligned}
$$




\section{RUC Upper Level Wind Vector RMS Errors}

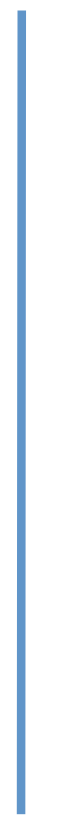

-We need a method to forecast wind uncertainty 